\title{
PASSIVE VIBRATION CONTROL OF AIRBORNE EQUIPMENT USING A CIRCULAR STEEL RING
}

\author{
Joseph Ellison', Goodarz Ahmadi ${ }^{1}$ and Mike Kehoe ${ }^{2}$
}

\begin{abstract}
Vibration isolation is needed to protect avionics equipment from adverse aircraft vibration environments. Passive isolation is the simplest means to achieve this goal. The system used here consists of a circular steel ring with a lump mass on top and exposed to base excitation. Sinusoidal and filtered zero-mean Gaussian white noise are used to excite the structure and the acceleration response spectra at the top of the ring are computed. An experiment is performed to identify the natural frequencies and modal damping of the circular ring. Comparison is made between the analytical and experimental results and good agreement is observed. The ring response is also evaluated with a concentrated mass attached to the top of the ring. The effectiveness of the ring in isolating the equipment from base excitation is studied. The acceleration response spectra of a single degree of freedom system attached to the top of the ring are evaluated and the results are compared with those exposed directly to the base excitation. It is shown that a properly designed ring could effectively protect the avionics from possible damaging excitation levels.
\end{abstract}

\footnotetext{
${ }^{1}$ Department of Mechanical and Aeronautical Engineering, Clarkson University, Potsdam, NY 13699-5725

${ }^{2}$ NASA Dryden Flight Research Facility, Edwards, CA 93523
} 


\section{Introduction}

Vibration control technology is used in many applications in order to protect equipment, structures or mechanisms from undesirable vibration environments. The isolator design is normally based on the degree of isolation desired and the frequency range of the disturbance. Munjal $^{1}$ has organized vibration isolators into groups according to their functions and Snowdon ${ }^{2}$ described the use and function of various isolators. Vibration isolation technology has been used in automobiles, aircraft, spacecraft and even buildings. Applications in the automobile are geared primarily toward improving ride quality. Aircraft design uses range from power plant, frictional damping of gas turbine blades ${ }^{3}$ and active engine mounts, to flutter suppression ${ }^{5}$. Agnes et al. ${ }^{6}$ described the vibration problems and solutions typical of fighter aircraft. Space applications vary in scope from structural control such as demonstrated by the INFLEX experiment ${ }^{7}$ to microgravity isolation ${ }^{8}$. Recently, application of vibration isolation to the Space Shuttle was studied by Lee-Glauser, et al. ${ }^{9}$ and the Space Station vibration control was discussed by Ellison, et al. ${ }^{10}$. In the area of earthquake engineering, frictional base isolation systems have been developed to protect large buildings (Mostaghel, et al. ${ }^{11}$, Su, et al. ${ }^{12}$ and Fan, et al. ${ }^{13}$ ).

Equipment mounted in aircraft are exposed to a varied vibration environment depending on the location of the installation and flight condition as illustrated by Dreher ${ }^{14}$. A survey of environmental data measured in flight such as vibration, acoustic, shock, thermal, flutter and loads was compiled by Hain et al. ${ }^{15}$ to define a "real-world" aircraft environment. One particular example is detailed in the vibration and acoustic measurements report on the F-111A aircraft ${ }^{16}$. The vibration environment directly influences the operation, performance and life expectancy of airborne equipment. To that end, test techniques were developed to evaluate the avionics equipment endurance based on the source of vibration, installation and response of the black box $^{17}$.

In order to reduce the environment at the equipment, use of vibration isolation has been suggested. A number of passive and active vibration control techniques are available and the choice depends on the degree of isolation that is desired or necessary ${ }^{18-21}$. Typical passive isolators consist of a resilient element contained in a metallic supporting frame. Generally, the resilient element is either an elastomer, air, steel spring, wire rope or metal mesh ${ }^{22}$. Wire rope isolators are highly effective in controlling both shock and vibration ${ }^{23}$. Passive isolators are usually capable of up to 80 percent isolation if the isolator natural frequency is less than onefourth of the lowest excitation frequency. Tuning of the isolator may be necessary to achieve such high levels of isolation ${ }^{24}$. Active control is used when additional protection is needed.

In this work, a passive isolation system is studied as a prelude to a combined active/passive system for protecting an avionics box subjected to base excitation. The base isolation system is composed of stainless steel circular rings placed on the base corners of the avionics box. This system has the capability of isolating the equipment in various directions. In this initial study, the avionics box is modeled as a lumped mass and only a single ring isolation system is considered. The earliest work performed on the free vibration of a circular ring was reported by Hoppe $^{25}$. Further work expanding the scope of the ring vibration problem considered centerline extension $^{26}$ and shear deformation and rotary inertia ${ }^{27-30}$. In most of the earlier works, the 
general ring vibration properties were analyzed but no particular vibration isolation applications were studied. Here, the ring is treated as a component of a passive isolation system and its performance in protecting the equipment against the adverse vibration environment is evaluated.

An experiment is also conducted to evaluate the vibration characteristics of a typical circular steel ring for comparison with the analysis. The experiment consisted of a circular steel ring mounted on top of a small electrodynamic shaker with the response measured by an accelerometer. The test is performed with and without an attached mass on top of the ring. This data is then used to verify the computational model.

Sinusoidal and random base excitations are considered and the peak responses of the ring and attached single degree of freedom systems are evaluated. It is shown that the ring could effectively reduce the peak acceleration transmitted to the avionics equipment.

\section{Model Formulation}

Passive base isolation of an avionics box with the use of circular, stainless steel ring supports attached to each corner of the equipment is shown schematically in Figure 1. Here, we assume that the avionics box is a rigid mass and consider the dynamics of a single ring. The basic configuration of this model, which consists of a thin stainless steel ring rigidly attached to a base structure and a concentrated mass located at the top, is shown in Figure 2.

Details of the derivation of Love's equation governing the vibration of a circular ring with a concentrated mass is outlined in Appendix A. Accordingly, the governing equations of motion for the tangential and the normal components of the ring displacement are given as

$$
\begin{gathered}
\frac{\partial^{6}}{\partial \theta^{6}} w_{a}(\theta, t)+2 \frac{\partial^{4}}{\partial \theta^{4}} w_{a}(\theta, t)+\frac{\partial^{2}}{\partial \theta^{2}} w_{a}(\theta, t)+\frac{a^{4}}{E I} \frac{\partial}{\partial \theta}\left[M_{\mathrm{p}} \frac{\partial^{3}}{\partial \theta \partial t^{2}} w_{a}(\theta, t)\right] \\
-\frac{M_{\rho} a^{4}}{E I} \frac{\partial^{2}}{\partial t^{2}} w_{a}(\theta, t)=\frac{a^{4}}{E I}\left(-q_{w}-\frac{\partial q_{u}}{\partial \theta}\right), \\
\frac{\partial}{\partial \theta} w_{a}(\theta, t)=-u_{a}(\theta, t),
\end{gathered}
$$

where $w_{a}$ is the tangential deflection, $u_{a}$ is the radial deflection, $\theta$ is the angle measured from the vertical, $t$ is time, $E I$ represents the bending stiffness, $a$ is the ring radius, $q_{w}$ is an external tangential force per unit length and $q_{u}$ is an external normal force per unit length. The mass distribution of the ring (including the concentrated mass located at the top of the ring) per unit length is given as ${ }^{31}$ 


$$
M_{\rho}=\rho A+\frac{m_{A}}{2 \pi a} \delta(\theta-\pi)
$$

where $\rho$ is the mass density of the ring, $A$ is the cross sectional area, $m_{A}$ is the mass of the avionics box represented by a concentrated mass located at $\theta=\pi$ and $\delta()$ denotes the Dirac delta function.

\section{Free Vibration of a Ring}

The natural frequencies of the ring are obtained by solving the homogenous form of Eq. (1) (in the absence of the concentrated mass at the top of the ring). Assuming a solution of the form ${ }^{28}$

$$
w_{a}(\theta, t)=W(\theta) e^{i \omega t}
$$

the resulting equation may be restated as

$$
\frac{d^{6} W}{d \theta^{6}}+2 \frac{d^{4} W}{d \theta^{4}}+\frac{d^{2} W}{d \theta^{2}}\left(1-\Omega^{2}\right)+\Omega^{2} W=0,
$$

where

$$
\Omega^{2}=\frac{\omega^{2}}{\omega_{o}{ }^{2}} \quad \text { and } \quad \omega_{o}{ }^{2}=\frac{E I}{\rho A a^{4}} .
$$

The appropriate boundary conditions are

$$
\left.\begin{array}{c}
W(\theta)=0 \\
-U(\theta)=\frac{d W(\theta)}{d \theta}=0 \\
\frac{d^{2} W(\theta)}{d \theta^{2}}=0
\end{array}\right\} \quad \text { at } \theta=0 \text { and at } \theta=2 \pi
$$

The solution to Eq. (5) is given by

$$
W(\theta)=\sum_{j=1}^{6} C_{j} e^{\lambda_{j} \theta}
$$

where the $C_{j}$ 's are found using the boundary conditions given by Eq. (7) and the $\lambda_{j}$ 's are the roots of the dispersion equation given as

$$
\lambda^{6}+2 \lambda^{4}+\lambda^{2}\left(1-\Omega^{2}\right)+\Omega^{2}=0 .
$$


When the boundary conditions are applied to the solution given by Eq. (8), a group of six simultaneous equations are obtained. In matrix form, these equations are expressed as

$$
\left[\begin{array}{cccccc}
1 & 1 & 1 & 1 & 1 & 1 \\
\lambda_{1} & \lambda_{2} & \lambda_{3} & \lambda_{4} & \lambda_{5} & \lambda_{6} \\
\lambda_{1}{ }^{2} & \lambda_{2}{ }^{2} & \lambda_{3}{ }^{2} & \lambda_{4}{ }^{2} & \lambda_{5}{ }^{2} & \lambda_{6}{ }^{2} \\
e^{2 \lambda_{1} \pi} & e^{2 \lambda_{2} \pi} & e^{2 \lambda_{3} \pi} & e^{2 \lambda_{4} \pi} & e^{2 \lambda_{5} \pi} & e^{2 \lambda_{6} \pi} \\
\lambda_{1} e^{2 \lambda_{1} \pi} & \lambda_{2} e^{2 \lambda_{2} \pi} & \lambda_{3} e^{2 \lambda_{3} \pi} & \lambda_{4} e^{2 \lambda_{4} \pi} & \lambda_{5} e^{2 \lambda_{5} \pi} & \lambda_{6} e^{2 \lambda_{6} \pi} \\
\lambda_{1}{ }^{2} e^{2 \lambda_{1} \pi} & \lambda_{2}{ }^{2} e^{2 \lambda_{2} \pi} & \lambda_{3}{ }^{2} e^{2 \lambda_{3} \pi} & \lambda_{4}{ }^{2} e^{2 \lambda_{4} \pi} & \lambda_{5}{ }^{2} e^{2 \lambda_{5} \pi} & \lambda_{6}{ }^{2} e^{2 \lambda_{6} \pi}
\end{array}\right]\left[\begin{array}{c}
C_{1} \\
C_{2} \\
C_{3} \\
C_{4} \\
C_{5} \\
C_{6}
\end{array}\right]=0 .
$$

A nontrivial solution for the $C_{j}$ 's exist only if the determinant of the coefficient matrix in (10) is zero.

An iterative procedure is used to find the natural frequencies and the corresponding modal coefficients. An initial guess for the frequency $(\Omega)$ is made and then the $\lambda_{j}$ 's are computed from Eq. (9). Using these $\lambda_{j}$ 's, the determinant of the coefficient matrix (10) is evaluated. If the determinant does not become zero, then the initial frequency guess is adjusted and the process is repeated until the determinant approaches zero. The mode shape coefficients, $\mathrm{C}_{\mathrm{i}}$, are then evaluated for each natural frequency using Eq. (10) and choosing $C_{6}=1$. The modal coefficients are then normalized for each mode such that

$$
\sqrt{\sum_{j=1}^{6} C_{j}^{2}}=1
$$

Using the dimensions and physical properties for the circular ring given in Table 1, Eqs. (9) and (10) are evaluated for $\Omega, \omega_{j}$ and $\lambda_{j}$. The modal frequencies and corresponding $\Omega_{j}$ 's are listed in Table 2. Due to the complex form of the $\lambda_{j}$ 's obtained, Eq. (8) may be stated as

$$
W_{k}(\theta)= \begin{cases}\sum_{i=1}^{3}\left(C_{2 i-1, k} e^{a_{i, \lambda} \theta} \cos b_{i, k} \theta+C_{2 i, k} e^{a_{i, k} \theta} \sin b_{i, k} \theta\right) & \text { for } \mathrm{k}=1,2,3, \\ \sum_{i=1}^{2}\left(C_{2 i-1, k} e^{a_{i, \lambda} \theta}+C_{2 i, k} e^{-a_{i, k} \theta}\right)+C_{5, k} \cos b_{3, k} \theta+C_{6, k} \sin b_{3, k} \theta \text { for } \mathrm{k}=4,5,6\end{cases}
$$

The mode shape coefficients and parameters for Eq. (12) that were found using the frequencies and $\lambda_{j}$ 's from Eqs. (9) and (10) are listed in Table 3 and the corresponding mode shapes are shown in Figure 3. The mode shapes are categorized according to their motion at the top of the ring $(\theta=\pi)$, either lateral or vertical. The odd numbered modes exhibit predominantly side to side motion and are thereby called lateral modes. Whereas the even numbered modes have 
mostly an up and down response and therefore are labeled vertical modes. Only the first six modes are shown and used in the calculations.

\section{Ring Response Due to Base Excitation}

In this section, the response of a circular ring, shown in Figure 2, subjected to base excitation is analyzed. The absolute motion of the ring is assumed to be given as

$$
w_{a}(\theta, t)=w(\theta, t)-y(t) \cos \theta+z(t) \sin \theta
$$

and

$$
u_{a}(\theta, t)=u(\theta, t)-y(t) \sin \theta-z(t) \cos \theta
$$

where $u$ and $w$ are relative, normal and tangential, ring displacements and $y$ and $z$ are base displacements.

The motion of the ring relative to the base is assumed to be given by

$$
w(\theta, t)=\sum_{k} \eta_{k}(t) W_{k}(\theta)
$$

and

$$
u(\theta, t)=\sum_{k} \eta_{k}(t) U_{k}(\theta)
$$

Substituting Eqs. (3), Eqs. (13) and (15) into Eq. (1) yields

$$
\begin{aligned}
\frac{\partial^{6}}{\partial \theta^{6}} \sum_{k} \eta_{k}(t) W_{k}(\theta)+2 \frac{\partial^{4}}{\partial \theta^{4}} \sum_{k} \eta_{k}(t) W_{k}(\theta)+\frac{\partial^{2}}{\partial \theta^{2}} \sum_{k} \eta_{k}(t) W_{k}(\theta) \\
+\frac{a^{4}}{E I} \frac{m_{a}}{2 \pi a} \delta^{\prime}(\theta-\pi)\left(\sum_{k} \ddot{\eta}_{k}(t) \frac{d W_{k}}{d \theta}+\ddot{y}(t) \sin \theta+\ddot{z}(t) \cos \theta\right) \\
+\frac{a^{4}}{E I}\left(\rho \mathrm{A}+\frac{m_{a}}{2 \pi a} \delta(\theta-\pi)\right)\left(\sum_{k} \ddot{\eta}_{k}(t) \frac{d^{2} W_{k}}{d \theta^{2}}-\sum_{k} \ddot{\eta}_{k}(t) W_{k}+2 \ddot{y}(t) \cos \theta-2 \ddot{z}(t) \sin \theta\right) \\
=\frac{a^{4}}{E I}\left(-q_{w}-\frac{\partial q_{k}}{\partial \theta}\right) .
\end{aligned}
$$

Using the relation given by Eq. (5) in Eq. (17) and simplifying yields

$$
\sum_{k}\left\{\left(\ddot{\eta}_{k}+\omega_{k}{ }^{2} \eta_{k}\right)\left(W_{k}-\frac{d^{2} W_{k}}{d \theta^{2}}\right)+\frac{m_{A}}{2 \pi \rho A a} \ddot{\eta}_{k}\left[\left(W_{k}-\frac{d^{2} W_{k}}{d \theta^{2}}\right) \delta(\theta-\pi)-\frac{d W_{k}}{d \theta} \delta^{\prime}(\theta-\pi)\right]\right\}
$$




$$
\begin{gathered}
=\frac{1}{\rho A}\left(q_{w}+\frac{\partial q_{u}}{\partial \theta}\right)+\left(1+\frac{m_{A}}{2 \pi \rho A a} \delta(\theta-\pi)\right)(2 \ddot{y}(t) \cos \theta-2 \ddot{z}(t) \sin \theta) \\
+\frac{m_{A}}{2 \pi \rho A a} \delta^{\prime}(\theta-\pi)(\ddot{y}(t) \sin \theta+\ddot{z}(t) \cos \theta) .
\end{gathered}
$$

Multiplying Eq. (18) by a normal mode, $W_{p}$, integrate around the ring and apply the orthogonality condition given by

$$
\int W_{p}\left(W_{k}-\frac{d^{2} W_{k}}{d \theta^{2}}\right) d \theta=0 \quad k \neq p
$$

we find

$$
\ddot{\eta}_{p}+2 \zeta_{p} \omega_{p} \dot{\eta}_{p}+\omega_{p}^{2} \eta_{p}+K_{p}=F_{p}+B_{p,} \ddot{y}(t)+B_{p_{z}} \ddot{z}(t)
$$

where

$$
\zeta_{p}=\frac{\gamma}{2 p A \omega_{p}}
$$

is the modal damping coefficient, $\gamma$ is an equivalent viscous damping factor introduced into Eq. $(20), \eta_{p}$ is the modal participation factor, $\omega_{p}$ is the $p^{\text {th }}$ natural frequency, $\ddot{z}(t)$ is the vertical base excitation and $\ddot{y}(t)$ is the lateral base excitation. The external pressure field is given by

$$
q_{w}=0 \quad \text { and } \quad q_{u}=\frac{m_{A} g}{2 \pi a} \delta(\theta-\pi)
$$

where $m_{A} g$ is the static weight at the top of the ring.

The coefficients in Eq. (20) are then defined as follows

$$
\begin{aligned}
& \mathrm{K}_{p}=\frac{m_{\mathrm{A}}}{2 \pi \rho A a N_{p}} \sum_{k} \ddot{\eta}_{k}\left(W_{k}(\pi) W_{p}(\pi)+\frac{d W_{k}(\pi)}{d \theta} \frac{d W_{p}(\pi)}{d \theta}\right) \\
& F_{p}=-\frac{m_{\mathrm{A}} g}{2 \pi \rho A a N_{p}} \frac{d W_{p}(\pi)}{d \theta} \\
& B_{p_{p}}=\frac{1}{N_{p}}\left(2 \int_{\theta} W_{p}(\theta) \cos \theta d \theta-\frac{m_{\mathrm{A}}}{2 \pi \rho A a} W_{p}(\pi)\right) \\
& B_{p_{z}}=\frac{1}{N_{p}}\left(-2 \int_{\theta} W_{p}(\theta) \sin \theta d \theta+\frac{m_{\mathrm{A}}}{2 \pi \rho A a} \frac{d W_{p}(\pi)}{d \theta}\right)
\end{aligned}
$$


where

$$
N_{p}=\int_{\theta}\left(W_{p}^{2}(\theta)-\frac{d^{2} W_{p}(\theta)}{d \theta^{2}} W_{p}(\theta)\right) d \theta
$$

is the norm of the $\mathrm{p}^{\text {th }}$ modal amplitude.

The equations given in Eq. (20) are coupled since $\mathrm{K}_{\mathrm{p}}$ depends on $\sum_{k} \ddot{\eta}_{k}(t)$. Here, a Gaussian reduction scheme is used to solve the equations simultaneously for the modal participation factors in the numerical simulation.

\section{Excitations}

Both sinusoidal and random base excitations are used to evaluate the performance of the passive vibration isolation system. Sinusoidal base accelerations applied are given by

$$
\ddot{y}(t)=Y_{a} \sin \left(\omega_{e} t\right) \quad \text { and } \quad \ddot{z}(t)=Z_{a} \sin \left(\omega_{e} t\right)
$$

where $Y_{a}$ and $Z_{a}$ are the amplitudes, $\omega_{e}$ is the excitation frequency and $t$ is time. The base accelerations, $\ddot{y}(t)$ and $\ddot{z}(t)$, are used in the following analyses.

The random excitation being used is modeled after NASA Ames/Dryden Flight Research Facility (ADFRF) process specification No. 21-2 for environmental testing of electronic and electromechanical equipment ${ }^{32}$, as shown in Figure 4. The random excitation model uses the frequency sampling method to design a nonrecursive finite impulse response (FIR) filter. As illustrated in Figure 5, zero-mean Gaussian white noise, $\varepsilon(t)$, is input to the filter (with impulse response $h(t))$, and the output being the random excitation, $\ddot{y}(t)$ or $\ddot{z}(t)$. That is

$$
\ddot{y}(t)=\int_{0}^{t} h(t-\tau) \varepsilon_{y}(\tau) d \tau \quad \text { and } \quad \ddot{z}(t)=\int_{0}^{t} h(t-\tau) \varepsilon_{z}(\tau) d \tau
$$

The mean and autocorrelation of the stationary, Gaussian white noise processes, $\varepsilon_{y}(t)$ and $\varepsilon_{z}(t)$, used as the input are given as:

$$
\begin{aligned}
& \left\langle\varepsilon_{y}(t)\right\rangle=0,\left\langle\varepsilon_{y}\left(t_{1}\right) \varepsilon_{y}\left(t_{2}\right)\right\rangle=2 \pi S_{o,} \delta\left(t_{1}-t_{2}\right),\left\langle\varepsilon_{y}\left(t_{1}\right) \varepsilon_{z}\left(t_{2}\right)\right\rangle=0, \\
& \left\langle\varepsilon_{z}(t)\right\rangle=0,\left\langle\varepsilon_{z}\left(t_{1}\right) \varepsilon_{z}\left(t_{2}\right)\right\rangle=2 \pi S_{o_{z}} \delta\left(t_{1}-t_{2}\right)
\end{aligned}
$$


where the angular brackets, ' \langle\rangle$\rangle$, represent ensemble averaging and $S_{o y}$ and $S_{o_{z}}$ are the constant power spectral intensities. The spectral intensity of the white noise excitations for the $y$ and $z$ directions are assumed to be given as

$$
S_{o_{y}}=.0043 \mathrm{~g}^{2} \mathrm{sec} \quad S_{o_{z}}=.0218 \mathrm{~g}^{2} \mathrm{sec}
$$

The value for $S_{o_{\varepsilon}}$ was estimated so that the spectral density of the vertical random excitation would fit that of the NASA ADFRF process specification. The lateral value was taken to be onefifth the vertical value since the lateral excitation intensity is usually much lower than that of the vertical excitation in aircraft. The impulse response, $h(t)$, is obtained by inverse Fourier transform given as

$$
h(t)=\mathfrak{J}^{-1}[H(k)]
$$

where $H(k)$ is the system function. The system function is a function of the acceleration power spectral density function represented in Figure 4 and given by

$$
|H(k)|=\sqrt{\frac{N}{2 \Delta t} \tilde{G}(k)}
$$

where $N$ is the number of samples, $\Delta \mathrm{t}$ is the spacing between time samples and $\tilde{G}(k)$ is the power spectral density function.

To design a FIR filter with the frequency response shown in Figure 4, the frequency response is sampled $N$ times at intervals of $k F_{s} / N, k=0,1, \ldots, N-1$ where $F_{s}$ is the sampling frequency. Since these values are related to the Fourier transform of the filter impulse response, the filter coefficients $h(n) \quad[\equiv h(n \Delta t)]$ are found using an inverse Fourier transform of the frequency samples from Eq. (33). For linear phase filters with a positive symmetrical impulse response, it is possible to rewrite the inverse Fourier transform such that the FIR filter coefficients are given by $^{33}$

$$
h(n)=\frac{1}{N}\left[\sum_{k=1}^{N / 2-1} 2|H(k)| \cos \mid[2 \pi k(n-\alpha) / N]+H(0)\right]
$$

where $H(k)$ are the frequency samples, $n=0, \ldots, N-1$ and $\alpha=(N-1) / 2$. Here $\Delta t=0.1$ sec and $N=1024$ samples are used. The impulse response of the above FIR filter is shown in Figure 6.

The Fast Fourier Transform (FFT) is used to transform the filter coefficients and white noise to the frequency domain. They are multiplied together and the result is inverse transformed using the inverse FFT back to the time domain. This produces time samples representative of the frequency response shown in Figure 4. A sample simulated excitation time history is plotted in Figure 7 . To verify that the procedure truly generates the appropriate frequency response, an 
ensemble of 1000 sample time histories were generated, transformed to the frequency domain and the averaged spectrum, $\frac{1}{\mathrm{~T}}|\vec{z}(\omega)|^{2}$, was evaluated. The resulting frequency response is shown in Figure 8. Comparing Figure 4 and Figure 8, it is observed that the filter produces time samples representative of the desired excitation spectrum.

\section{Experiment}

The purpose of the experiment was to measure the natural frequencies and damping of a circular steel ring for comparison with the analytical results. The experimental set-up is shown schematically in Figure 9. The dimensions and physical properties of the circular ring that was used in the experiment are the same as those listed in Table 1 and used in the analysis. Two 5 $\mathrm{mm}$ holes were drilled in the ring, one at the top and one at the bottom. The ring was bolted directly to the shaker armature through the bottom hole for both the vertical and lateral vibration testing. For rings with the concentrated mass test conditions, a steel weight was bolted through the hole at the top of the ring. Accelerometer mounting wax was used to hold the accelerometer onto the ring. The accelerometer was mounted on top of the ring for measuring vertical modes of vibration and on the side of the ring for measuring lateral modes. Five test conditions were evaluated for various concentrated masses. The concentrated masses used were 0 grams, 130 grams, 250 grams, 430 grams and 780 grams.

The test system that was used to perform the experiment was entirely PC based. An A-D/D-A interface on the PC allowed for the computer to output the shaker drive signal and monitor the system response channels. Software was used for FFT processing and vibration analysis. Broadband random excitation from 0 to $2000 \mathrm{~Hz}$ was generated by the computer and used to shake the ring vertically. A force transducer was installed between the ring and shaker rod in order to measure the input excitation. An accelerometer was mounted $n$ top of the ring to measure the vertical acceleration. The accelerometer and force data were recorded using the PC based data acquisition system. Twenty five samples were acquired and averaged to produce the frequency response functions (FRF) for the vertical excitation for each test condition.

The lateral modes were measured using impact excitation. A small hammer was used to impact the side of the ring. An accelerometer mounted on the opposite side of the ring measured the ring response due to the impact. The response was recorded on the PC. Ten impact samples were measured and averaged and used to compute the power spectra for the lateral vibration data for each test condition. The experimental FRF's and power spectra were curve fit at each resonance frequency and the half-power bandwidth method was used to evaluate the modal damping. For a ring with no concentrated mass, the natural frequency and modal damping for the first six modes of the ring are listed in Table 4. For each of the test conditions with the concentrated mass attached to the ring, the two lowest modes are listed. Comparing Table 2 and Table 4 , it is observed that the computed natural frequencies are in close agreement with the experimental ones. 


\section{Results}

Computer simulation of the ring response is performed by solving Eqs. (13)-(16) and Eq. (20) subjected to random base excitations. A typical circular ring with the dimensions and physical properties given in Table 1 is analyzed. The ring fundamental frequency is $13.75 \mathrm{~Hz}$ with a damping ratio of 0.036 . Two ring configurations, one without any concentrated mass and one with a $0.45 \mathrm{~kg}$ mass added on top of the ring, are examined. The ring deflection is given by

$$
\mathrm{u}=\mathrm{u}_{\mathrm{s}}+\mathrm{u}_{\mathrm{e}} \text {, }
$$

where $u_{s}$ is the static deflection under the weight and $u_{e}$ is the deflection relative to the static equilibrium. In this section, the vibration of the ring about the static equilibrium is studied. The static deflection, $u_{s}$, results from the gravitational force term given by Eq. (24). To achieve this in the numerical simulation, Eq. (20) is evaluated in the absence of base excitation. i.e.,

$$
\eta_{p}=\frac{F_{p}}{\omega_{p}^{2}} .
$$

This equation is evaluated and the modal participation factors are recorded. These values then establish the static equilibrium position of the ring due to the concentrated mass and can be calculated using Eqs. (2), (12), (16) and

$$
u_{s}(\theta, t)=\sum_{p} \eta_{p}(t) U_{p}(\theta) .
$$

Subsequent analyses are then conducted using this static equilibrium position as a starting point. The stored values are used as initial conditions for solving Eq. (20) under base excitation.

Using both sinusoidal and random excitations, the acceleration at the top of the ring and the response of a single degree of freedom (SDOF) system attached to the top of the ring are evaluated. The peak responses of the ring are computed for a range of ring frequencies. To assess the effectiveness of the base isolation at the point on the ring where the equipment is attached, acceleration response spectra are evaluated. The lateral and vertical acceleration response spectra are respectively defined as

$$
\left.S_{a}\left(\omega_{a}, \zeta_{a}\right)=\max _{t}\left\{\ddot{w}_{a}(\pi, t)\right\}\right\}
$$

and

$$
S_{a}\left(\omega_{a}, \zeta_{a}\right)=\max _{t}\left\{\ddot{u}_{a}(\pi, t)\right\} .
$$

For random excitation, the mean, the standard deviation, the absolute maximum and the absolute minimum acceleration are defined, respectively, as: 


$$
\begin{aligned}
& \bar{a}\left(\omega_{a}, \zeta_{a}\right)=\left\langle S_{a}\left(\omega_{a}, \zeta_{a}\right)\right\rangle, \\
& \sigma_{a}\left(\omega_{a}, \zeta_{a}\right)=\left\langle\left(S_{a}\left(\omega_{a}, \zeta_{a}\right)-\bar{a}\right)^{2}\right\rangle^{1 / 2}, \\
& a_{\max }\left(\omega_{a}, \zeta_{a}\right)=\max \left\{S_{a}\left(\omega_{a}, \zeta_{a}\right)\right\}, \\
& a_{\min }\left(\omega_{a}, \zeta_{a}\right)=\min \left\{S_{a}\left(\omega_{a}, \zeta_{a}\right)\right\} .
\end{aligned}
$$

It should be noted that the absolute maximum and minimum accelerations, which represent upper and lower bounds on the simulation results, are sample dependent.

\section{Comparison of Numerical and Experimental Results}

\section{Ring without concentrated mass}

In order to verify the validity of the numerical model, the analytical ring response is computed in the same manner as was done in the experiment. Random excitation given by Eq. (29) is used to excite the ring in both lateral and vertical directions. The response at the top of the ring is computed and used with the input base excitation to generate vertical and lateral frequency response functions as shown in Figure 10 and Figure 11, respectively. The experimental frequency response functions are also plotted in these figures for comparison. The peaks in each frequency response function correspond to the natural frequencies of the ring as listed in Table 2. The damping values that were calculated from the experimental data as listed in Table 4 were used in the analytical model.

Figure 10 and Figure 11 show reasonable general agreement between the experimental and numerical frequency response functions. However, the amplitude of the peak values at each resonant frequency are not in agreement between experiment and analysis. Part of the difference in peak amplitude may be due to the frequency response of the accelerometer used. The accelerometer is rated for an operating range of 10-10000 $\mathrm{Hz}$. The accelerometer frequency response is level down to about $100 \mathrm{~Hz}$. However, the response starts to roll off below $100 \mathrm{~Hz}$ and the accelerometer is unusable below about $10 \mathrm{~Hz}$. Therefore the amplitude of the experimental resonant frequencies below $100 \mathrm{~Hz}$ are attenuated for both the vertical and lateral results. The peak response amplitudes of the lateral results can not be compared directly since the experimental results were acquired with impact excitation producing power spectra density functions and the numerical results were generated with random excitation giving frequency response functions.

\section{Ring With Rigid Avionics}

Next, the circular ring with a 0.45 kilogram rigid avionics mass attached to its top is analyzed. The ring response is computed in the same manner as outlined above for Figure 10 and Figure 11. Random excitation given by Eq. (29) is used to excite the ring in both the lateral and vertical directions. Vertical and lateral frequency response functions are plotted in Figure 12 and 13. 
Experimental frequency response functions are also shown in these figures for comparison. The ring geometry is the same as that used earlier with the exception of the additional mass. Comparing Figure 10 and 11 with Figure 12 and 13, it is noticed that the ring natural frequencies are lower with the additional mass on top as expected. Both experimental and numerical results indicate a decrease in the natural frequencies with fairly close agreement between the two. The observed variations in the resonant frequency peak amplitudes are due to accelerometer frequency response characteristics and for the lateral case, are also due to the different methods of excitation used. Numerical results for the lateral case are produced with random excitation whereas impact excitation is used in the experiment. Figure 12 and 13 show close agreement in overall trends between the experimental and numerical data.

\section{Avionics Box Under Sinusoidal Excitation}

In this section, responses of a SDOF avionics box subject to horizontal-vertical sinusoidal base excitation with and without a ring isolation system are studied. Figure 14 illustrates the system configurations considered. Sinusoidal base excitation, as given by Eq. (28), is used. The amplitude, $A$, of the input excitation is $0.01 \mathrm{~g}$ 's in the vertical direction and $0.002 \mathrm{~g}$ 's in the lateral direction. The excitation frequency, $\mathrm{f}_{e}$, is $400 \mathrm{~Hz}$. The peak responses of the system are computed for a range of avionics equipment system frequencies from 50 to $500 \mathrm{~Hz}$. Table 1 lists the physical properties of the ring used in the analysis with the natural frequencies that appear in Table 2. The interaction of the avionics system and the ring is neglected in the following analyses since it is assumed that the avionics system is rigidly attached to the ring.

The vertical and lateral acceleration response spectra of the avionics system are plotted in Figure 15 and Figure 16. The cases of unprotected avionics and avionics with the ring are shown. For the avionics with the ring, two different responses are shown. No interaction implies that there was no coupling between the avionics and ring degrees of freedom. The response at the top of the ring was computed and used as excitation to the SDOF avionics box. The rigid mass response implies that the avionics box is treated as a rigid mass of $0.45 \mathrm{~kg}$. Both figures show a peak at the excitation frequency of $400 \mathrm{~Hz}$ due to the resonance of the SDOF system with the excitation frequency. The ring natural frequencies given in Table 2 are not excited by the sinusoidal excitation as shown by the plots. For the vertical direction, there is a $50 \%$ decrease in the transmitted acceleration to the avionics system on top of the ring with no interaction mass and an order of magnitude decrease for the rigid mass case as compared to the avionics system exposed directly to the sinusoidal excitation, unprotected avionics. Similar decreases are seen for the transmitted acceleration in the lateral direction. There is an order of magnitude decrease for the avionics system on the ring with no interaction mass and a one and a half order of magnitude decrease when the avionics on top of the ring is treated as a rigid mass. Clearly, the ring provides significant passive isolation for the avionics box.

\section{Ring Response to Random Excitation}

For the case of a single ring (in the absence of a concentrated attached mass), the vertical ring response spectra for the top of the ring are plotted versus ring frequency in Figure 17 and the lateral response spectra are plotted in Figure 18. The peak responses for an ensemble of 100 
samples are computed using the random excitation given by Eq. (29) and the results are compiled and the statistical response spectra are evaluated from Eqs. (38)-(43). The input power spectra density level is $0.04 \mathrm{~g}^{2} / \mathrm{Hz}$ up to $150 \mathrm{~Hz}$ and then increases at $4 \mathrm{DB} / \mathrm{Oct}$ to $0.10 \mathrm{~g}^{2} / \mathrm{Hz}$ at $300 \mathrm{~Hz}$ as shown in Figure 4 and Figure 8. Six modes are included in the numerical ring model. The mean vertical acceleration spectrum starts at about $0.002 \mathrm{~g}$ for a ring frequency of $15 \mathrm{~Hz}$ and increases over the frequency range plotted leveling off to about $0.025 \mathrm{~g}$ at a frequency of about $150 \mathrm{~Hz}$. The mean lateral vibration starts off at about $0.0003 \mathrm{~g}$ at the lower frequencies (about 5 $\mathrm{Hz}$ ) and steadily increases to about $0.003 \mathrm{~g}$ at a frequency of $60 \mathrm{~Hz}$. This increase in acceleration is a result of the higher frequency modes responding to the increasing excitation levels The maximum and minimum acceleration levels are an extreme upper and lower bound, respectively, for the computed vibration spectrum.

To evaluate the effects of damping on ring performance, the vertical ring acceleration response spectra as a function of structural damping are calculated and plotted in Figure 19. The other ring parameters are as given in Table 1. The results are as expected, indicating a decrease in transmitted vibration with an increase in structural damping. It is observed from the graph that a damping value of about six percent provides satisfactory isolation. For a passive ring system having six percent damping as compared to that with a two percent damping, there is a $36 \%$ decrease in transmitted excitation. Whereas another four percent increase in damping above six percent only provides an additional $14 \%$ decrease in the transmitted vibration. Increasing the damping in the ring system above ten percent provides little if any improvement in the vibration environment at the top of the ring.

\section{Avionics Under Random Excitation}

For the random base excitation, comparison is made between the acceleration responses of the avionics system mounted on top of the ring (treated as a rigid $0.45 \mathrm{~kg}$ concentrated mass) and the unprotected avionics system. The system response is computed for a range of avionics system frequencies from 50 to $500 \mathrm{~Hz}$. The sensitivity to variations in the ring damping ratio on the response spectra is also examined. The resulting response spectra are plotted versus the avionics system frequency. As was noted before, the avionics system is treated as a rigid concentrated mass attached to the ring and the interaction between the avionics system and the ring is neglected.

The vertical and lateral avionics system acceleration response spectra with and without the ring are plotted in Figure 20 and Figure 21. Comparing the response of the isolated avionics to that of the unprotected avionics, the transmitted vibration to the avionics system is cut in half using the ring as a passive isolator. When the avionics system is rigidly mounted on top of the ring, the acceleration levels are magnified at the ring natural frequencies as compared to the rest of the acceleration response. However, these levels are still considerably lower than the acceleration levels for the unprotected system. Even the maximum calculated acceleration levels of the isolated system are less than half the unprotected avionics minimum response. In the lateral direction, the ring isolates the avionics system by at least an order of magnitude over the entire frequency range. The isolated avionics response again is greater at the ring resonant frequencies 
as compared to the rest of the spectrum. The ring performs better in the lateral direction by not transmitting the lateral excitation into the avionics system as readily as the vertical excitation.

The peak acceleration response of the avionics system rigidly mounted to the ring is calculated for several damping ratios and plotted as a function of avionics frequency in Figure 22 and Figure 23. These curves are compared to the response of the unprotected avionics system. The default damping values referred to in the figures are those listed in Table 2 with the $100 \%$ value being twice what is listed and $-50 \%$ being half the listed value. As shown in both figures, the peak acceleration decreases with increasing structural damping. Again, the results are as expected. Increasing the ring damping increases the isolation effectiveness of the passive ring element and the peak acceleration levels for all damping values are less than the levels for the unprotected system.

\section{Conclusions}

An analytical model of a stainless steel circular ring for the purpose of base isolation of avionics equipment is formulated. This model is used to evaluate the circular ring response characteristics subject to base excitation. Filtered zero-mean Gaussian white noise was tuned to match the NASA process specification No. 21-2 and is used for the random excitation. Peak acceleration response spectra are evaluated as a function of avionics frequency and ring structural damping. The ring is evaluated as an passive isolator for a rigidly mounted avionics system. This system is compared to the response of the unprotected system. An experiment was performed to measure the modal response of the thin circular steel ring mounted on top of an electrodynamic shaker. The analytical ring responses closely match the experimental results. The peak avionics system acceleration spectra indicate that the ring is an effective isolator in both the vertical and lateral directions.

The sensitivity of the ring response and avionics system response to variations in ring parameters and additional mass on top of the ring is examined. With no added mass at the top of the ring, the peak ring acceleration increases with increasing excitation. Changes in structural damping produce expected results in peak ring acceleration; decreasing transmitted acceleration with increasing damping factor. Overall, the ring and avionics system response were insensitive to small variations in ring properties.

\section{Acknowledgments}

This work is supported by the NASA Dryden Flight Research Facility, Edwards, CA under the Grant No. NGT-51314. 


\section{References}

${ }^{1}$ Munjal, M.L., A Rational Synthesis of Vibration Isolators, Journal of Sound and Vibration, Vol. 39, pp. 247-263, 1975.

${ }^{2}$ Snowden, J.C., Vibration Isolation Use and Characterization, Journal of the Acoustical Society of America, Vol. 66, pp. 1245-1279, 1979.

${ }^{3}$ Plunkett, R., Damping Applications for Vibration Control, Friction Damping, ASME Booklet, AMD-38, 1980.

${ }^{4}$ Sumali, H. and Cudney, H.H., An Active Engine Mount with a Piezoelectric Stacked Actuator, 35th Structures, Structural Dynamics, and Materials Conference, AIAA-94-1466-CP, April 1994.

${ }^{5}$ Triplett, W.E., Kappus, H.F. and Landy, R.J., Active Flutter Suppression Systems for Military Aircraft: A Feasibility Study, Air Force Flight Dynamics Laboratory, AFFDL-TR-72-116, 1973.

${ }^{6}$ Agnes, G.S., Whitehouse, S.R. and Mackaman J.R., Vibration Attenuation of Aircraft Structures Utilizing Active Materials, Proceedings of the Smart Structures and Materials Conference, Vol. 1917(1), pp. 368-379, Feb. 1993.

${ }^{7}$ Gogan, R.E., INFLEX Final Report, Harris Corporation, Melbourne, FL, Dec. 1992.

${ }^{8}$ Knospe, C.R., Hampton, R.D. and Allaire, P.E., Control Issues of Microgravity Vibration Isolation, Acta Astronautica, Vol. 25(11), pp. 687-697, Nov. 1991.

${ }^{9}$ Lee-Glauser, G. and Ahmadi, G., Dynamic Response Spectra for an Aerospace Payload and Its Attachment, Clarkson Report No. MIE-232, 1991.

${ }^{10}$ Ellison, J., Ahmadi, G. and Grodsinsky, C., Evaluation of Passive and Active Vibration Control Mechanisms in a Microgravity Environment, J. of Spacecraft and Rockets, Vol. 32, No. 2, pp. 375-376, March 1995.

"Mostaghel, N. and Tanbakuchi, J., Response of Sliding Structures to Earthquake Support Motion, Earthquake Engng. Struct. Dyn., Vol. 11, pp. 729-748, 1983.

${ }^{12}$ Su, L., Ahmadi, G. and Tadjbakhsh, I.G., A Comparative Study of Base Isolation Systems, J. of the Engng. Mechanics Division, ASCE, Vol. 115, pp. 1976-1992, 1989.

${ }^{13}$ Fan, F. and Ahmadi, G., A , J. , 19xx.

${ }^{14}$ Dreher, J.F., Aircraft Equipment Random Vibration Test Criteria Based on Vibrations Induced By Turbulent Airflow Across Aircraft External Surfaces, Shock and Vibration Bulletin, Bulletin 43, Part 3, pp. 127-139, June 1973.

${ }^{15}$ Hain, R.F., Coronado, A.R. and Dillard, M., Bibliography of Environmental Data Measured in Flight, Air Force Flight Dynamics Laboratory, AFFDL-TR-93-XXX, 1993.

${ }^{16}$ Fisher, C.P. and Price, R.G., Vibration and Acoustic Measurements on F-111A Number 75, Clean Airplane in Level Flight, General Dynamics Corporation, FZS-12-321, March 1971.

${ }^{17}$ Wafford, J.H., Application of MIL-STD-810C Dynamic Requirements to USAF Avionics Procurements, AGARD Report No. 682, pp. 1-1 -- 1-11, Nov. 1979.

${ }^{18}$ Geng, Z. and Haynes, L.S., 6-Degree-of-Freedom Active Vibration Isolation Using a Stewart Platform Mechanism, Journal of Robotic Systems, Vol. 10(5), pp. 725-744, 1993.

${ }^{19}$ Huba, A., Vibration Isolation of Hpt-Equipment, Mechatronics, Vol. 37(2), pp. 204-205, 1991.

${ }^{20}$ Chen, H.M. and Lewis, P., Adaptive Control for a Vibration Isolation Mount, Trans. of the ASME, Noise, Control and Acoustics, Vol. 8, pp. 121-124, 1990.

${ }^{21}$ Nelson, P.G., An Active Vibration Isolation System for Inertial Reference and Precision Measurement, Review of Scientific Instruments, Vol. 62(9), pp. 2069-2075, 1991. 
${ }^{22}$ Gilbert, C. and LeKuch, H., Isolating Shock and Vibration, Mechanical Engineering Journal, pp. 58-63, Oct. 1982.

${ }^{23}$ Tinker, M.L. and Cutchins, M.A., Damping Phenomena in a Wire Rope Vibration Isolation System, Journal of Sound and Vibration, Vol. 157(1), pp. 7-18, 1992.

${ }^{24}$ Stech, D.J., $\$ H_{-}\{2\} \$$ Approach for Optimally Tuning Passive Vibration Absorbers to Flexible Structures, J. Guidance, Vol. 17(3), pp. 636-638, 1993.

${ }^{25}$ Hoppe, R., The Bending Vibrations of a Circular Ring, Crelle Journal of Mathematics, Vol. 73 , pp. 158, 1871.

${ }^{26}$ Philipson, L.L., On the Role of Extension in the Flexural Vibrations of Rings, Journal of Applied Mechanics, Vol. 23, pp. 364-366, 1956.

${ }^{27}$ Seidel, S. and Erdely, E.A., On the Vibration of a Thick Ring in its Own Plane, Journal of Engineering for Industry, Vol. 86, pp. 240, 1964.

${ }^{28}$ Rao, S.S. and Sundararajan, V., In-plane Flexural Vibrations of Circular Rings, Journal of Applied Mechanics, Vol. 91, pp. 620-625, Sept. 1969.

${ }^{29}$ Rao, S.S., Effects of Transverse Shear and Rotatory Inertia on the Coupled Twist-Bending Vibrations of Circular Rings, Journal of Sound and Vibration, Vol. 16, pp. 551-566, 1971.

${ }^{30}$ Kirkhope, J., Simple Frequency Expression for the In-plane Vibration of Thick Circular Rings, Journal of the Acoustical Society of America, Vol. 59, pp. 86-89, 1976.

${ }^{31}$ Ahmadi, G. and Satter, M.A., On the Random Vibration of a Damped Simply Supported Beam Carrying Concentrated Masses, Industrial Mathematics, Vol. 24(1), pp. 19-27, 1974.

${ }^{32}$ NASA Ames-Dryden Flight Research Facility, Environmental Testing: Electronic and Electromechanical Equipment, Process Specification No. 21-2.

${ }^{33}$ Ifeachor, E.C. and Jervis, B.W., Digital Signal Processing: A Practical Approach, AddisonWesley Publishing Co., NY, 1993.

${ }^{34}$ Soedel, Werner, Vibrations of Shells and Plates, 2nd ed., Marcel Dekker, Inc., NY, 1993. 


\section{Appendix}

Love's equations of motion governing the vibrations of a circular ring in its plane of curvature due to an applied pressure field are ${ }^{34}$

$$
\frac{E I}{a^{4}}\left(\frac{\partial^{2} w(\theta, t)}{\partial \theta^{2}}-\frac{\partial^{3} u(\theta, t)}{\partial \theta^{3}}\right)+\frac{E A}{a^{2}}\left(\frac{\partial^{2} w(\theta, t)}{\partial \theta^{2}}+\frac{\partial u(\theta, t)}{\partial \theta}\right)+q_{w}=M_{\rho} \frac{\partial^{2} w(\theta, t)}{\partial t^{2}}
$$

$$
\frac{E I}{a^{4}}\left(\frac{\partial^{3} w(\theta, t)}{\partial \theta^{3}}-\frac{\partial^{4} u(\theta, t)}{\partial \theta^{4}}\right)-\frac{E A}{a^{2}}\left(\frac{\partial w(\theta, t)}{\partial \theta}+u(\theta, t)\right)+q_{u}=M_{\mathrm{p}} \frac{\partial^{2} u(\theta, t)}{\partial t^{2}}
$$

In most applications, the extension of the neutral surface of the ring is negligible and the inextensional approximation may be used. i.e.,

$$
\frac{\partial w(\theta, t)}{\partial \theta}=-u(\theta, t)
$$

The ring equations of motion need to be expressed in force and moment resultant form in order to apply the inextensional approximation. These equations are as follows

$$
\frac{1}{a} \frac{\partial}{\partial \theta} N_{\theta \theta}+\frac{1}{a^{2}} \frac{\partial}{\partial \theta} M_{\theta \theta}-M_{\mathrm{p}} \frac{\partial^{2}}{\partial t^{2}} w(\theta, t)=-q_{w}
$$

$$
\frac{1}{a^{2}} \frac{\partial^{2}}{\partial \theta^{2}} M_{\theta \theta}-\frac{N_{\theta \theta}}{a}-M_{\rho} \frac{\partial^{2}}{\partial t^{2}} u(\theta, t)=-q_{u}
$$

where $N_{\theta \theta}$ is the force and $\mathrm{M}_{\theta \theta}$ is the bending moment. Solving Eq. (A5) for $N_{\theta \theta}$ and substituting into Eq. (A4) gives

$$
\frac{1}{a^{2}} \frac{\partial^{3}}{\partial \theta^{3}} M_{\theta \theta}+\frac{1}{a^{2}} \frac{\partial}{\partial \theta} M_{\theta \theta}-M_{\mathrm{p}} \frac{\partial^{2}}{\partial t^{2}} w(\theta, t)-\frac{\partial}{\partial \theta}\left[M_{\mathrm{\rho}} \frac{\partial^{2}}{\partial t^{2}} u(\theta, t)\right]=-q_{w}-\frac{\partial q_{u}}{\partial \theta} .
$$

The resultant moment for the ring based on the tangential and normal displacements has the form

$$
M_{\theta \theta}=\frac{E I}{a^{2}}\left(\frac{\partial}{\partial \theta} w(\theta, t)-\frac{\partial^{2}}{\partial \theta^{2}} u(\theta, t)\right) .
$$


Using the inextensional approximation, Eq. (A7) can be expressed in terms of the tangential displacement as

$$
M_{\theta \theta}=\frac{E I}{a^{2}}\left(\frac{\partial}{\partial \theta} w(\theta, t)+\frac{\partial^{3}}{\partial \theta^{3}} w(\theta, t)\right)
$$

(A8)

or in terms of the normal displacement as

$$
M_{\theta \theta}=\frac{E I}{a^{2}}\left(u(\theta, t)+\frac{\partial^{2}}{\partial \theta^{2}} u(\theta, t)\right) .
$$

(A9)

Substituting Eqs. (A3) and (A8) into Eq. (A6) gives the equation for the tangential component of the ring motion as follows

$$
\begin{gathered}
\frac{\partial^{6}}{\partial \theta^{6}} w_{a}(\theta, t)+2 \frac{\partial^{4}}{\partial \theta^{4}} w_{a}(\theta, t)+\frac{\partial^{2}}{\partial \theta^{2}} w_{a}(\theta, t)+\frac{a^{4}}{E I} \frac{\partial}{\partial \theta}\left[M_{\mathrm{\rho}} \frac{\partial^{3}}{\partial \theta \partial t^{2}} w_{a}(\theta, t)\right] \\
-\frac{M_{\rho} a^{4}}{E I} \frac{\partial^{2}}{\partial t^{2}} w_{a}(\theta, t)=\frac{a^{4}}{E I}\left(-q_{w}-\frac{\partial q_{u}}{\partial \theta}\right)
\end{gathered}
$$

The normal component of the ring motion is obtained from Eqs. (A3) and (A10). 


\section{Tables}

Table 1: Ring Properties

\begin{tabular}{ll}
\hline Modulus, E & $199 \times 10^{2} \mathrm{~N} / \mathrm{m}^{2}$ \\
Poisson's Ratio, $\mu$ & 0.3 \\
Radius, a & $.07104 \mathrm{~m}$ \\
Thickness, h & $5.1 \times 10^{-4} \mathrm{~m}$ \\
Width, b & $.03165 \mathrm{~m}$ \\
Top mass, $\mathrm{m}$ & $.450 \mathrm{~kg}$ \\
Density, $\rho$ & $7916 \mathrm{~kg} / \mathrm{m}^{3}$ \\
\hline
\end{tabular}

Table 2: Analytical ring natural frequencies and damping values

\begin{tabular}{c|cccccc}
\hline Mode & 1 & 2 & 3 & 4 & 5 & 6 \\
\hline $\mathrm{f}_{\mathrm{j}}(\mathrm{Hz})$ & 13.75 & 37.50 & 78.75 & 133.75 & 202.50 & 282.50 \\
$\omega_{\mathrm{j}}(\mathrm{rad} / \mathrm{s})$ & 86.39 & 235.61 & 494.80 & 840.37 & 1272.34 & 1775.00 \\
$\Omega_{\mathrm{j}}$ & 0.56642 & 1.59520 & 3.38459 & 5.75492 & 8.68973 & 12.13275 \\
$\zeta_{\mathrm{j}}$ & 0.036 & 0.040 & 0.025 & 0.018 & 0.025 & 0.019 \\
\hline
\end{tabular}

Table 3: Ring mode shape coefficients and parameters.

\begin{tabular}{cccccccccc}
\hline Mode & $\mathrm{i}$ & \multicolumn{1}{c}{$\mathrm{a}_{\mathrm{i}}$} & \multicolumn{1}{c}{$\mathrm{b}_{\mathrm{i}}$} & \multicolumn{1}{c}{$\mathrm{C}_{\mathrm{i}}$} & Mode & $\mathrm{i}$ & \multicolumn{1}{c}{$\mathrm{a}_{\mathrm{i}}$} & \multicolumn{1}{c}{$\mathrm{b}_{\mathrm{i}}$} & \multicolumn{1}{c}{$\mathrm{C}_{\mathrm{i}}$} \\
\hline & 1 & 0.00 & 1.30876 & -0.10096 & & 1 & 0.00 & 1.68930 & -0.11704 \\
1 & 2 & 0.38027 & 0.53683 & -0.14731 & & 2 & 0.82800 & 0.50863 & -0.07916 \\
& 3 & -0.38027 & 0.53683 & -0.01967 & 2 & 3 & -0.82800 & 0.50863 & 0.00077 \\
& 4 & & & 0.03524 & & 4 & & & -0.00245 \\
& 5 & & & 0.12062 & & 5 & & & 0.11626 \\
& 6 & & & 0.42328 & & 6 & & & 0.45340 \\
& & & & & & & & & \\
& 1 & 0.00 & 2.17382 & -0.09447 & & 1 & 1.99490 & 0.00 & $0.79760 \mathrm{E}-6$ \\
& 2 & 1.20824 & 0.31161 & -0.05741 & & 2 & 1.07927 & 0.00 & -0.22151 \\
3 & 3 & -1.2082 & 0.31161 & 0.00033 & 4 & 3 & 0.00 & 2.67291 & -0.00033 \\
& 4 & & & 0.00018 & & 4 & & & 0.29688 \\
& 5 & & & 0.09413 & & 5 & & & -0.07503 \\
& 6 & & & 0.76404 & & 6 & & & -0.04531 \\
& & & & & & & & & \\
& 1 & 2.65515 & 0.00 & $-0.65433 \mathrm{E}-8$ & & 1 & 3.24700 & 0.00 & $0.11063 \mathrm{E}-9$ \\
& 2 & 1.02932 & 0.00 & -0.11509 & & 2 & 1.01428 & 0.00 & -0.08019 \\
5 & 3 & 0.00 & 3.17952 & 0.00027 & 6 & 3 & 0.00 & 3.68399 & -0.00022 \\
& 4 & & & 0.17655 & & 4 & & & 0.13268 \\
& 5 & & & -0.06173 & & 5 & & & -0.05225 \\
& 6 & & & -0.03904 & & 6 & & & -0.03409 \\
\hline
\end{tabular}


Table 4: Experimental ring natural frequencies and damping values.

\begin{tabular}{c|c|cccccc}
\hline $\begin{array}{c}\text { Mass } \\
\text { (grams) }\end{array}$ & Mode & 1 & 2 & 3 & 4 & 5 & 6 \\
\hline 0 & $\omega_{\mathrm{j}}$ & 86.394 & 229.336 & 507.367 & 854.513 & 1253.49 & 1790.71 \\
& $\zeta_{\mathrm{j}}$ & 0.036 & 0.040 & 0.025 & 0.018 & 0.025 & 0.019 \\
\hline 130 & $\omega_{\mathrm{j}}$ & 31.416 & 83.252 & & & & \\
& $\zeta_{\mathrm{j}}$ & 0.056 & 0.057 & & & & \\
\hline 250 & $\omega_{\mathrm{j}}$ & 21.991 & 69.115 & & & & \\
& $\zeta_{\mathrm{j}}$ & 0.112 & 0.036 & & & & \\
\hline 430 & $\omega_{\mathrm{j}}$ & 18.850 & 53.407 & & & & \\
& $\zeta_{\mathrm{j}}$ & 0.120 & 0.053 & & & & \\
\hline 780 & $\omega_{\mathrm{j}}$ & 12.566 & 39.270 & & & & \\
& $\zeta_{\mathrm{j}}$ & 0.091 & 0.060 & & & & \\
\hline
\end{tabular}




\section{Figures}

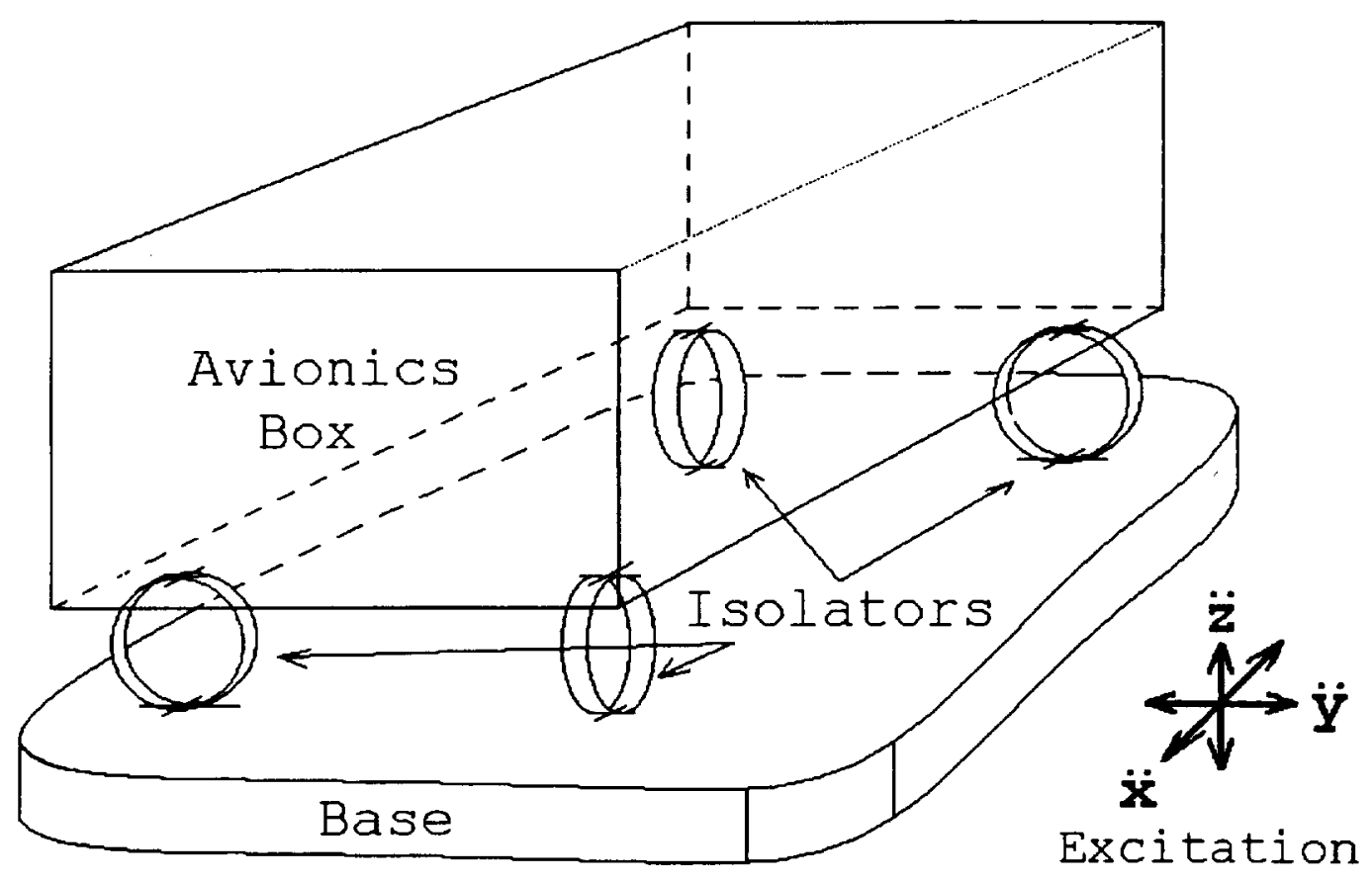

Figure 1: Circular ring isolation system for generic avionics equipment. .

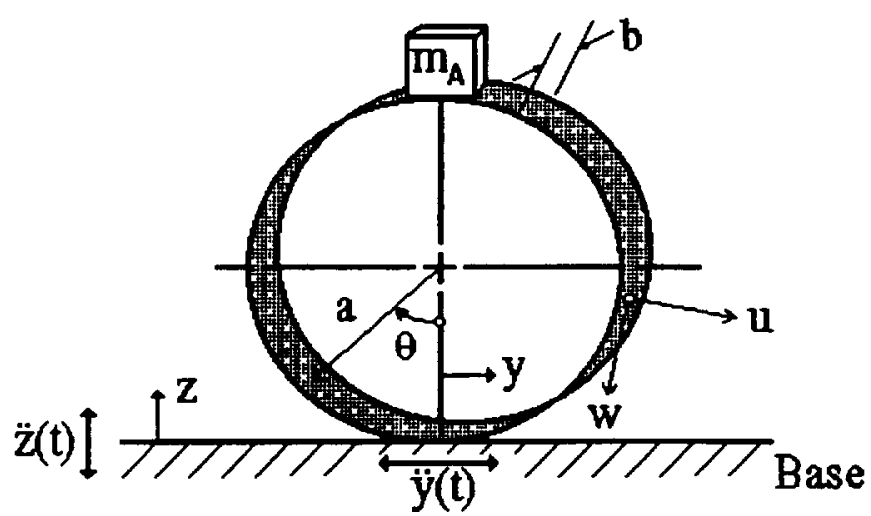

Figure 2: Circular ring structure. 

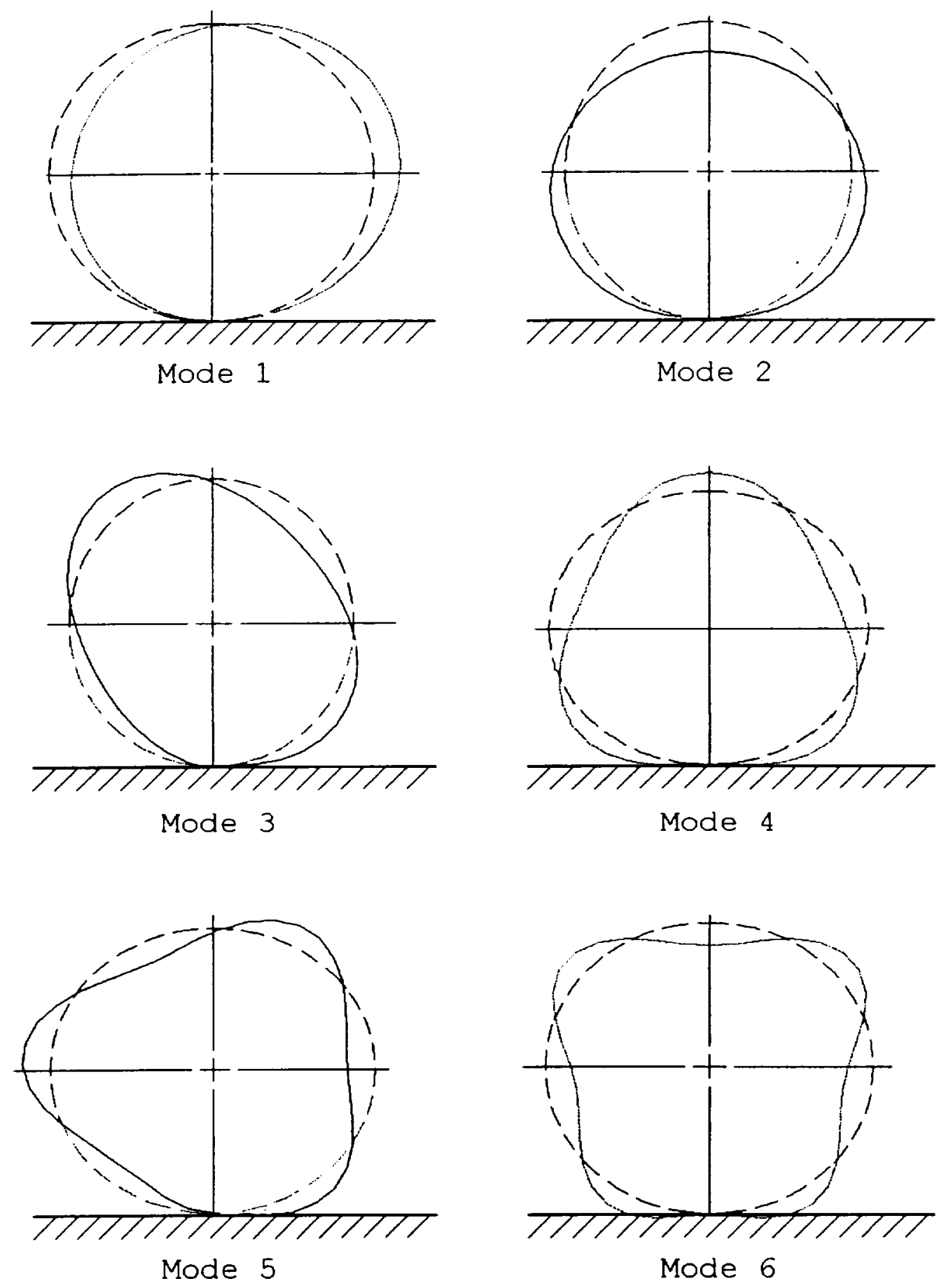

Figure 3: Circular ring mode shapes. 


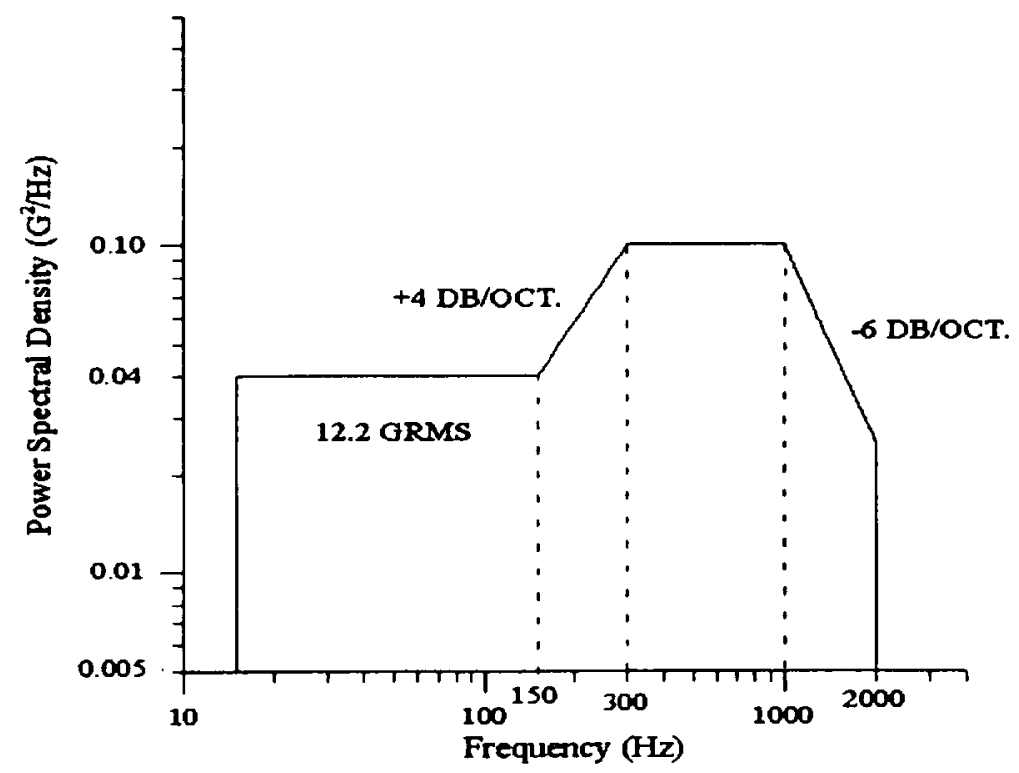

Figure 4: NASA ADFRF process specification No. 21-2.

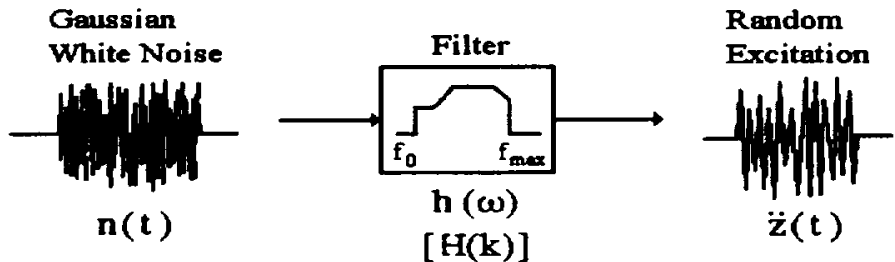

Figure 5: Random excitation model.

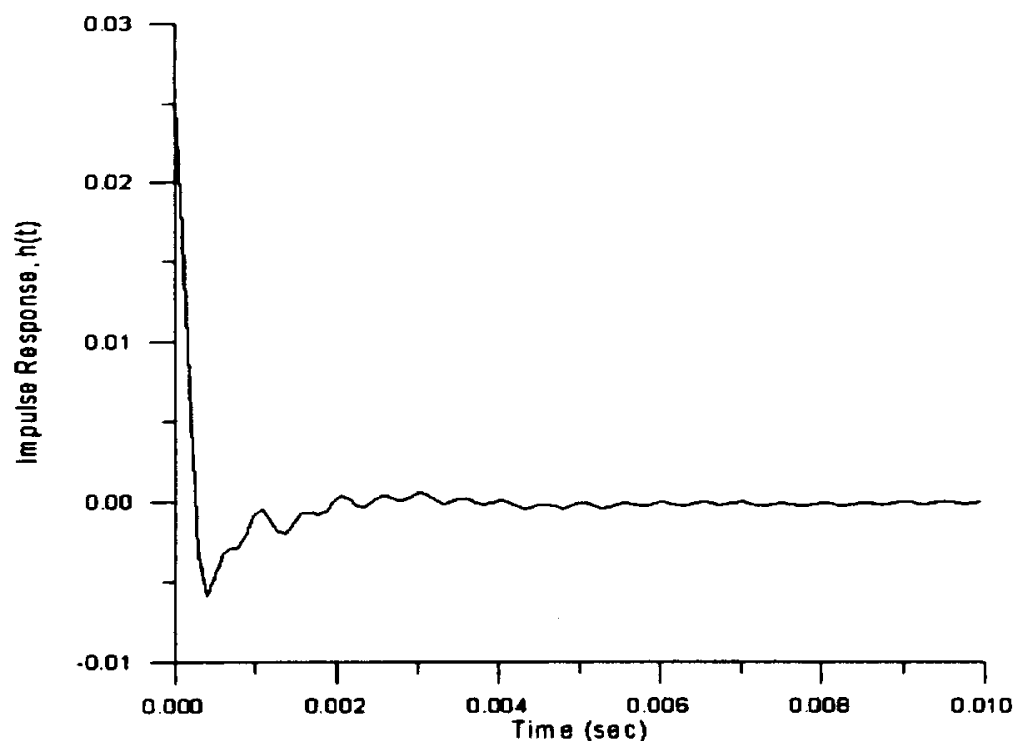

Figure 6: Impulse response function. 


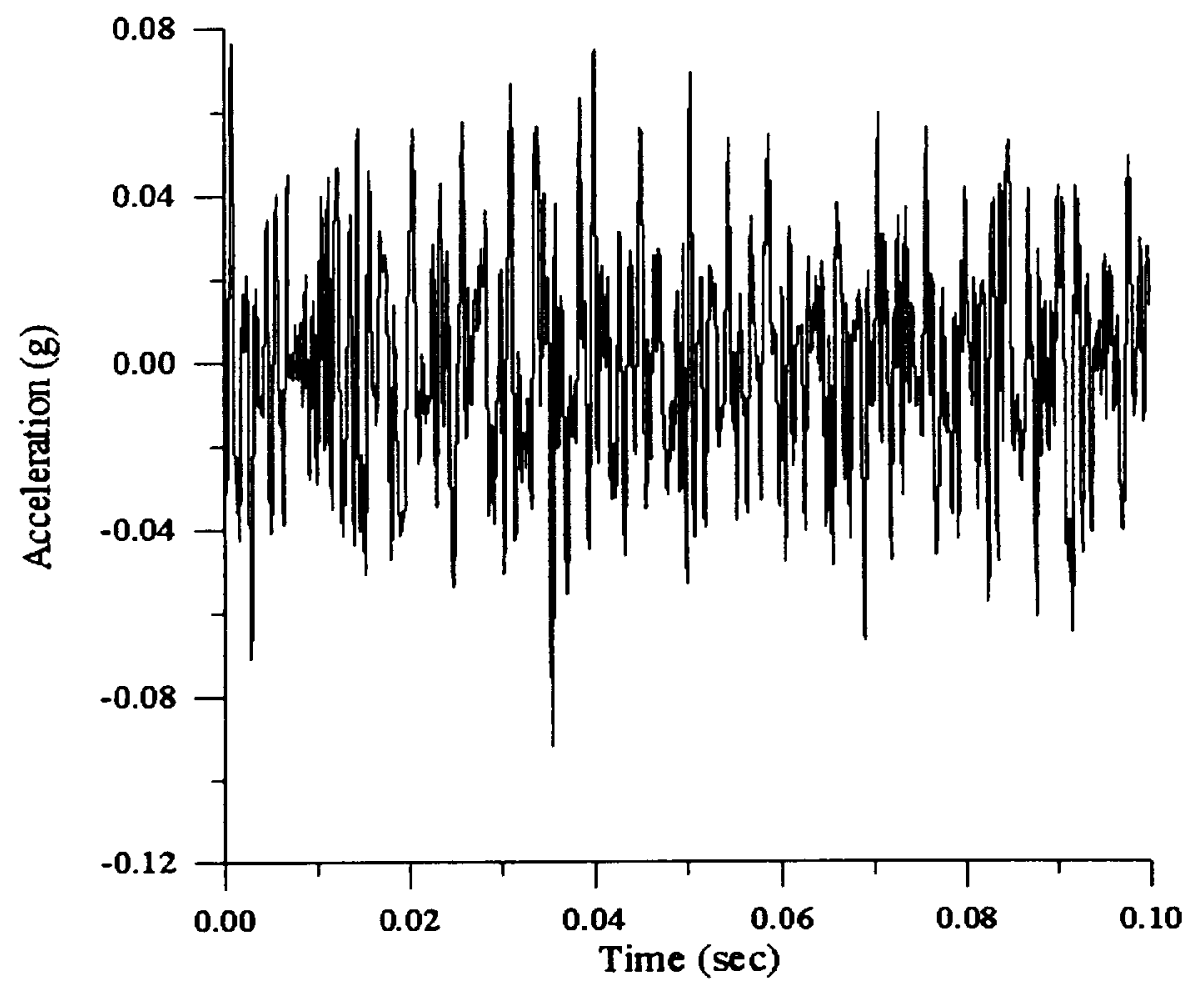

Figure 7: Sample excitation time history.

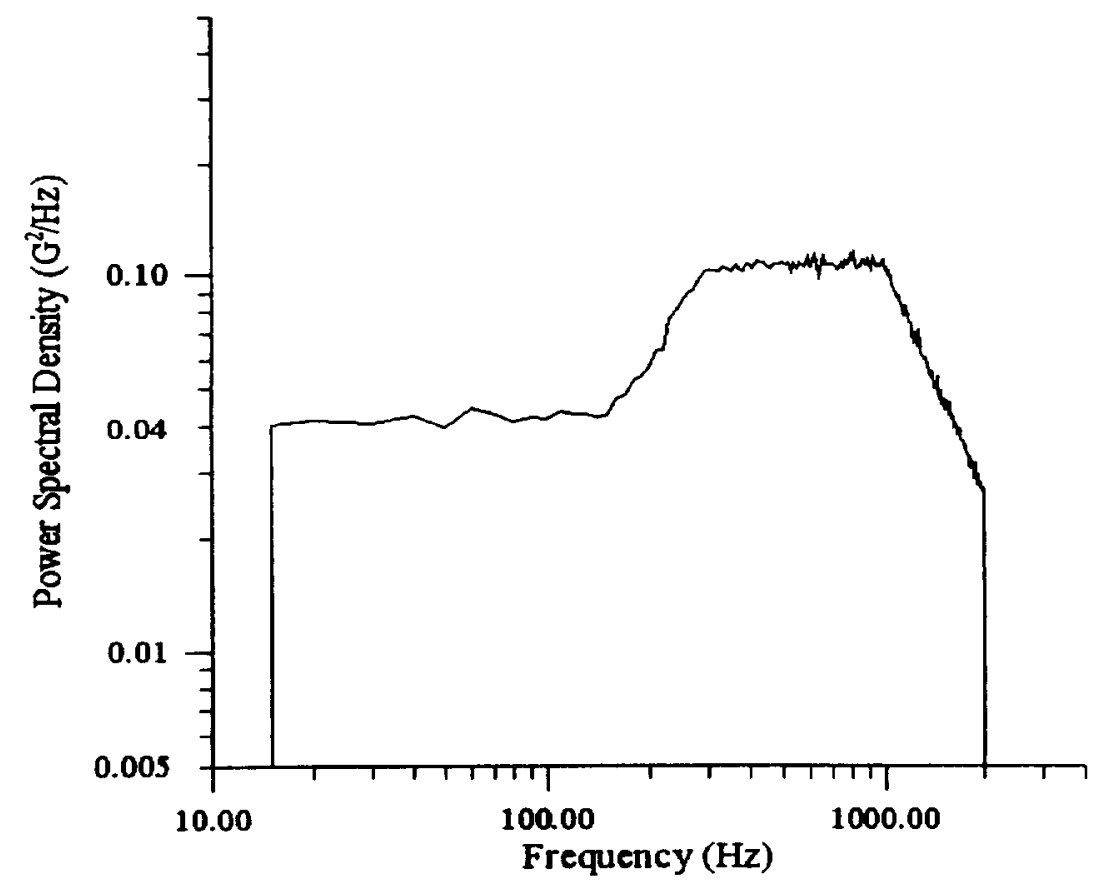

Figure 8: Frequency response of an ensemble average of 1000 time samples. 


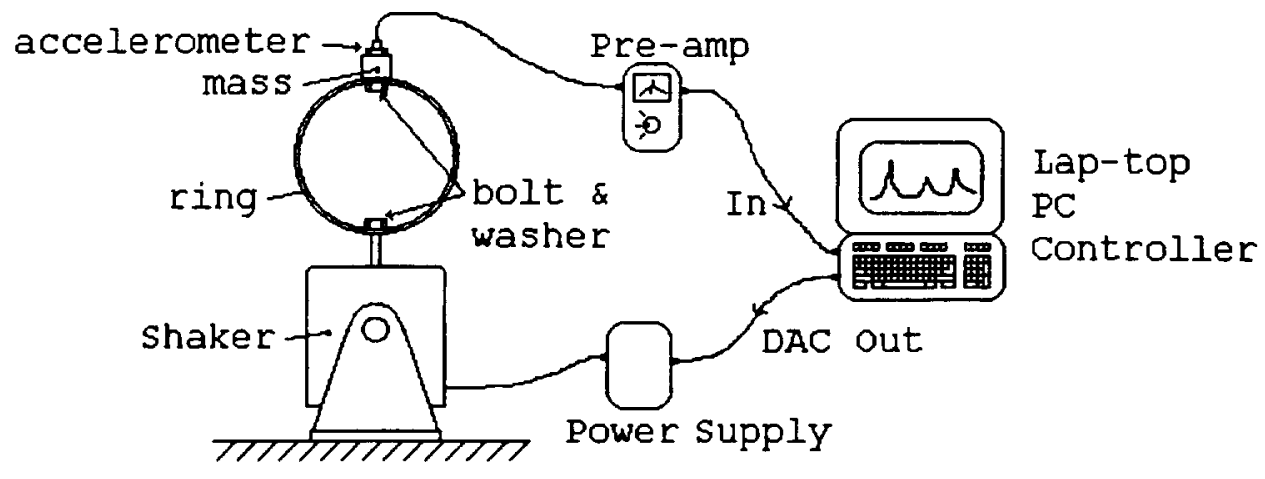

Figure 9: Experiment set-up for circular ring vibration.

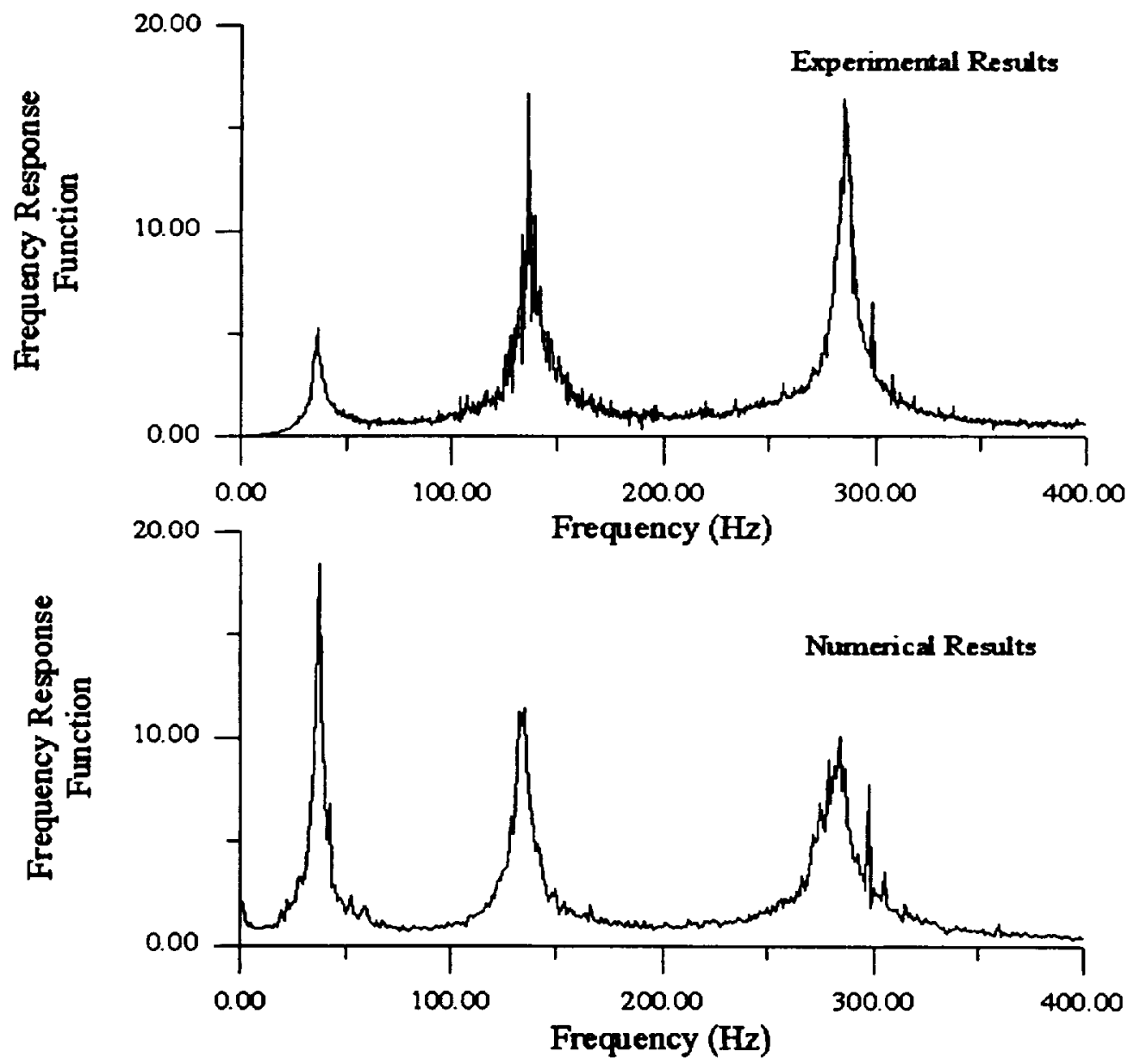

Figure 10: Comparison of analytical and experimental frequency response functions for the circular steel ring for vertical excitation. 


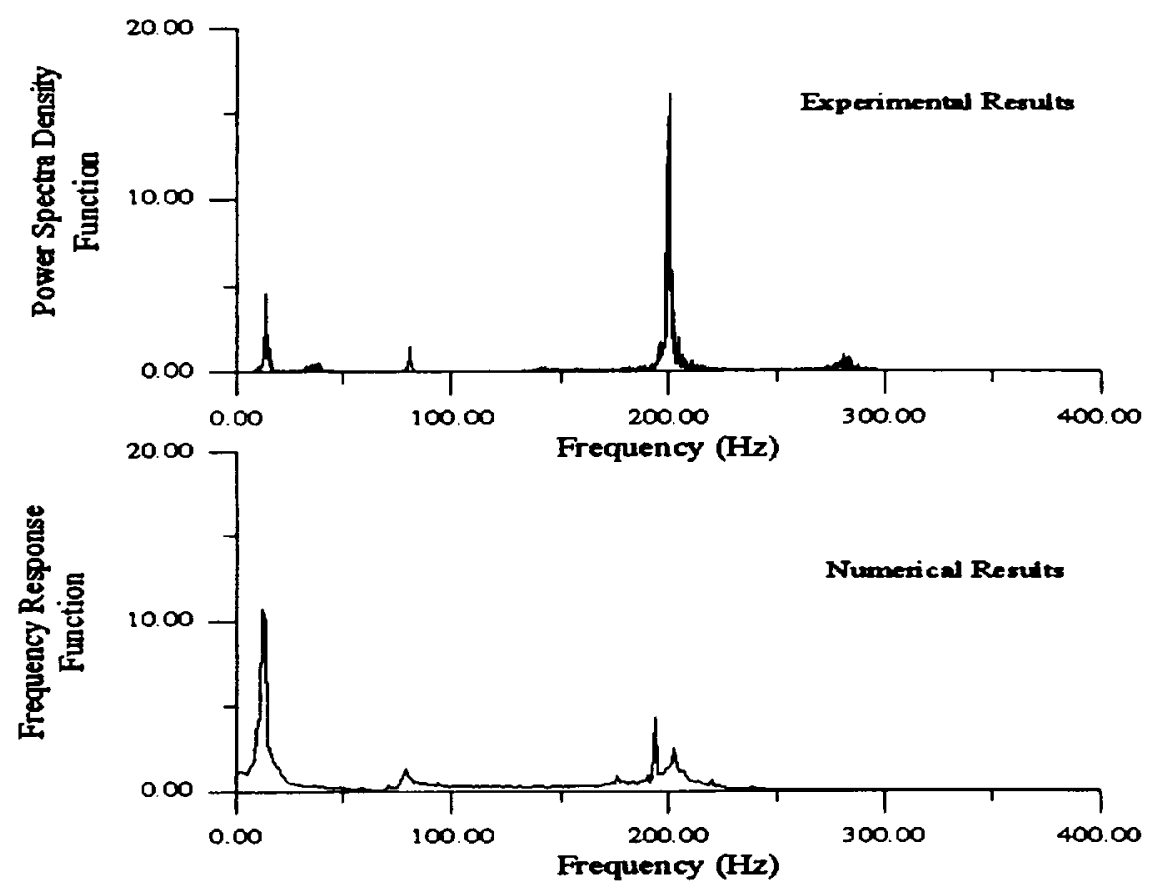

Figure 11: Comparison of analytical and experimental results for the circular steel ring for lateral excitation.

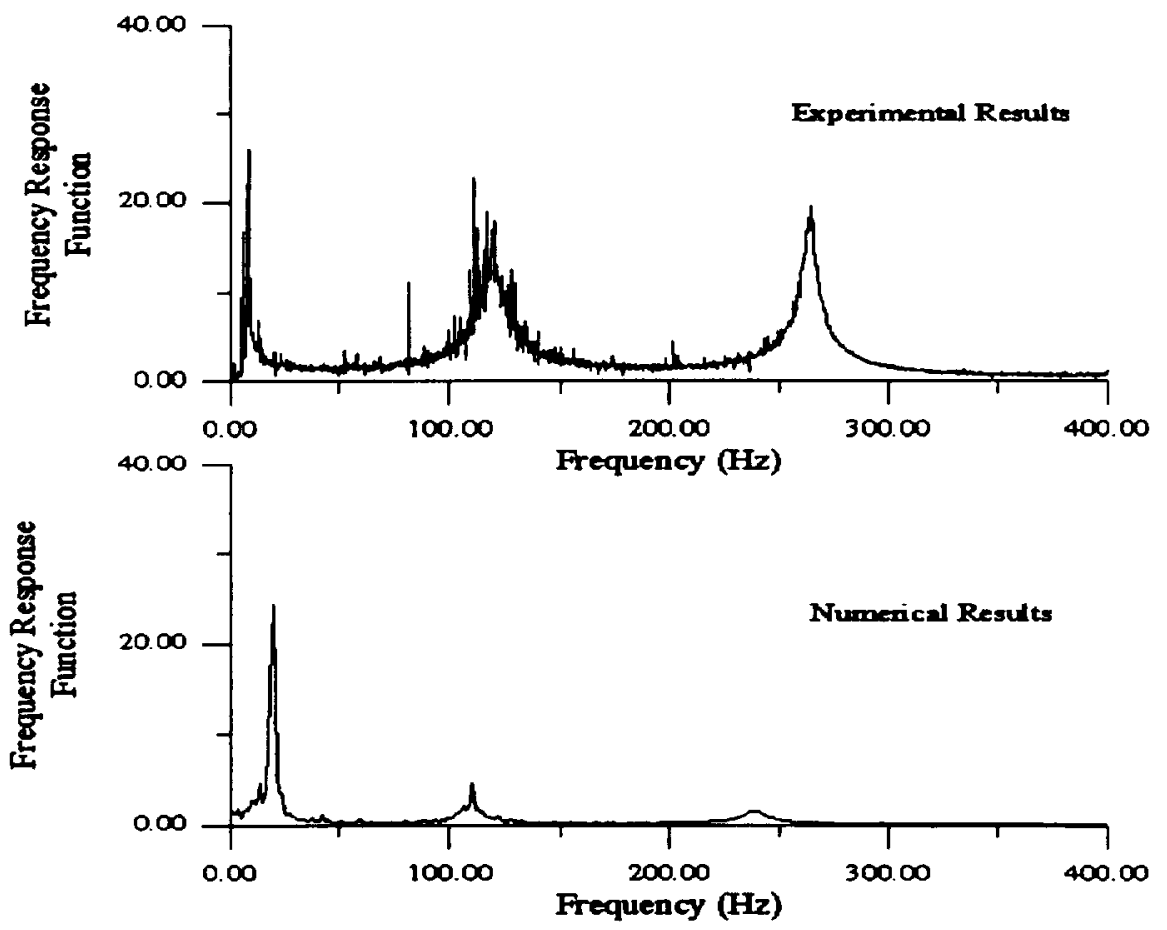

Figure 12: Comparison of analytical and experimental frequency response functions for the circular steel ring with a $0.45 \mathrm{~kg}$ concentrated mass under vertical random base excitation. 


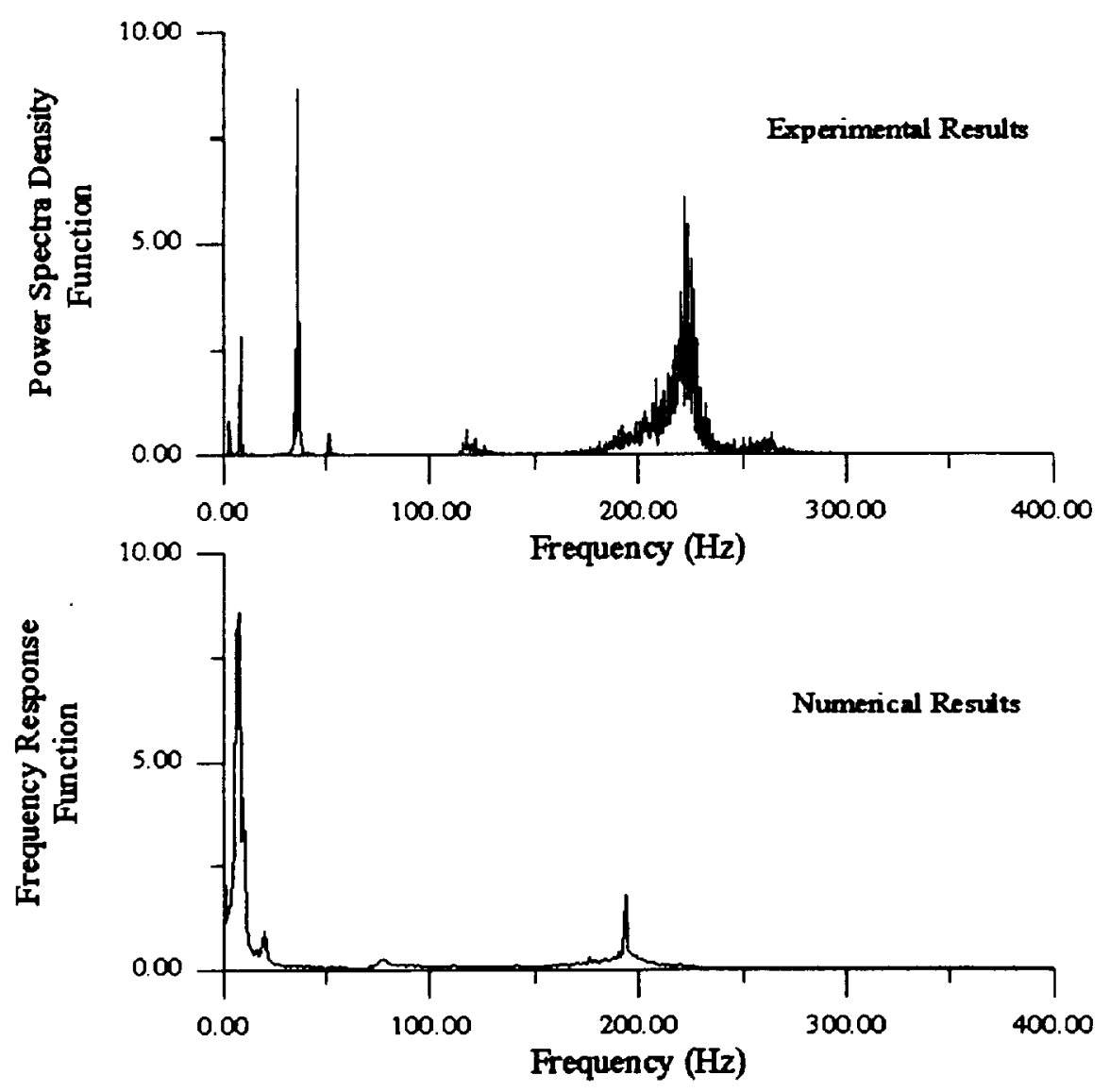

Figure 13: Comparison of analytical and experimental results for the circular steel ring with a $0.45 \mathrm{~kg}$ concentrated mass under lateral random base excitation.

(a)

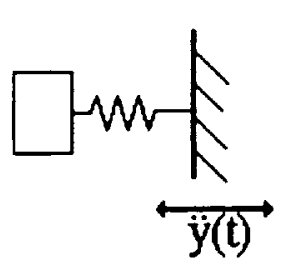

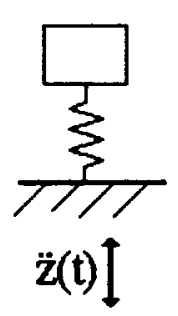

(b)

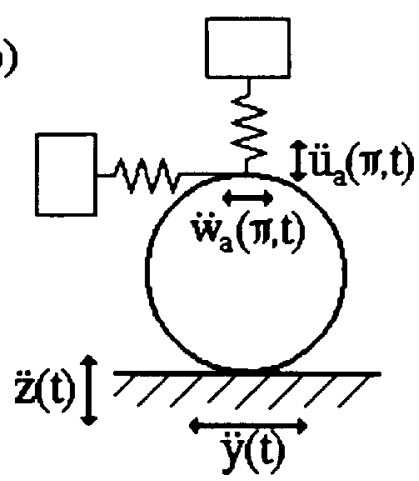

Figure 14: Single degree of freedom system configurations: (a) SDOF system exposed to random excitation without ring, (b) SDOF system exposed to transmitted vibration at top of ring. 


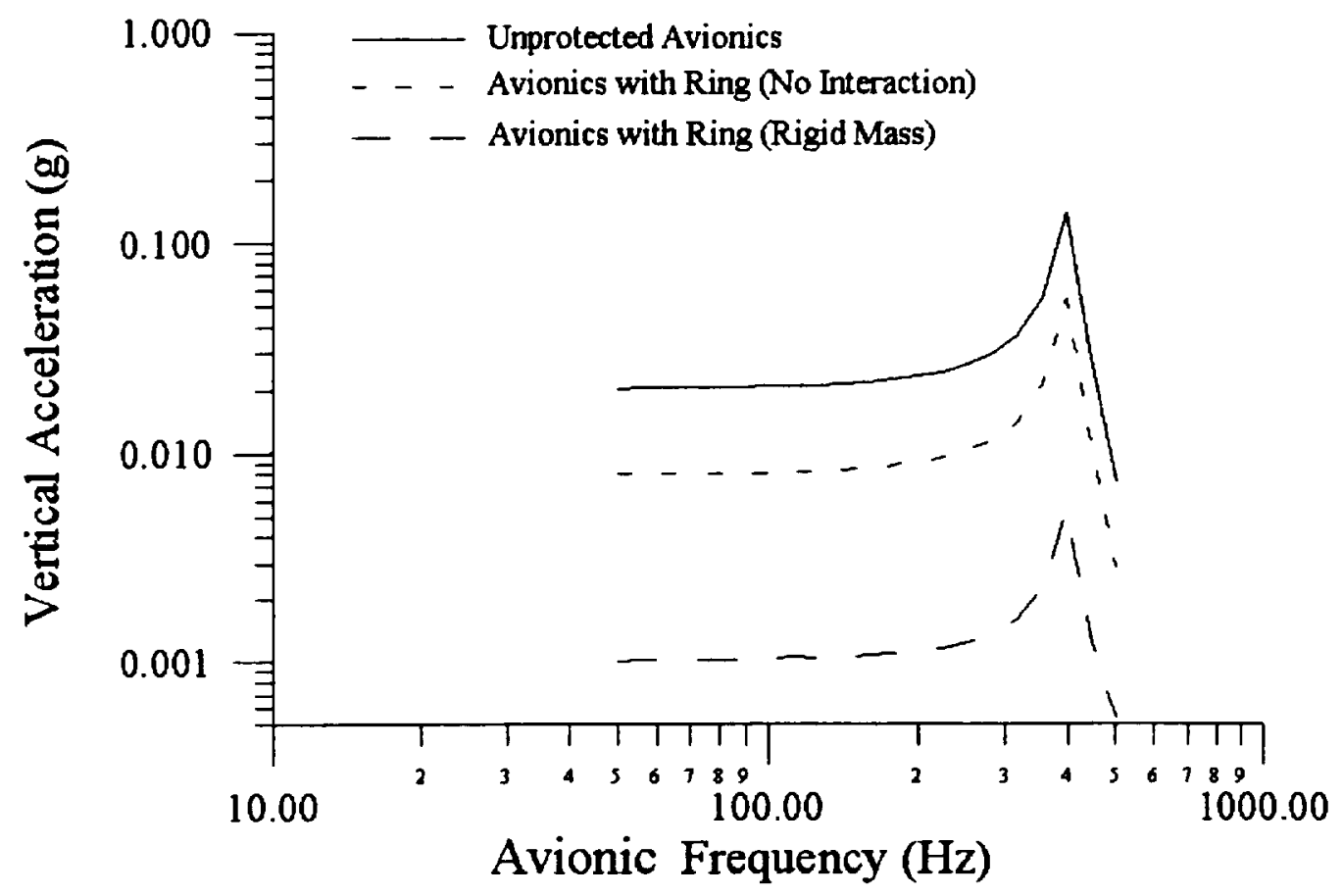

Figure 15: Comparison of vertical response spectra of an avionics system with and without the circular ring under sinusoidal base excitation.

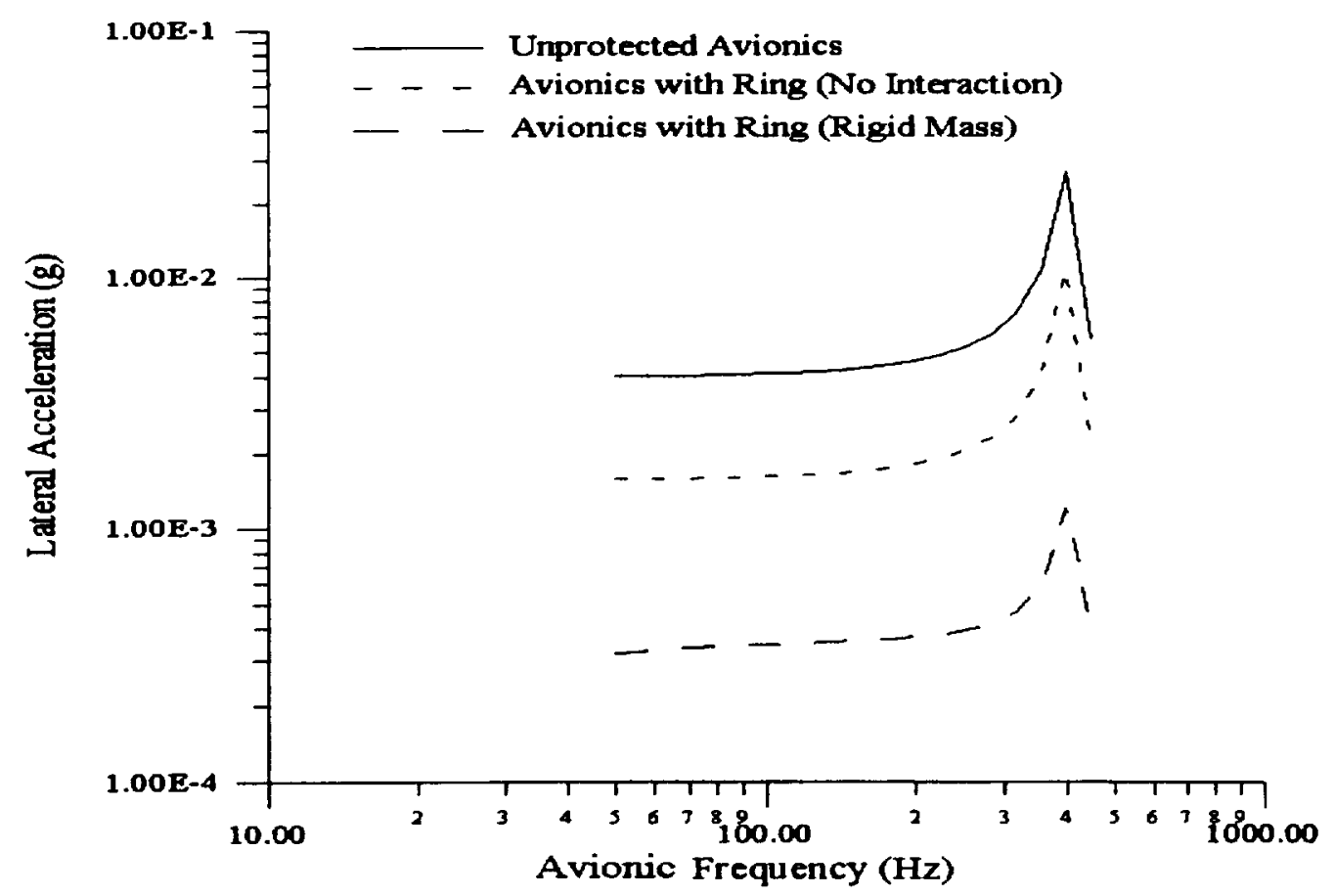

Figure 16: Comparison of lateral response spectra of an avionics system with and without the circular ring under sinusoidal base excitation. 


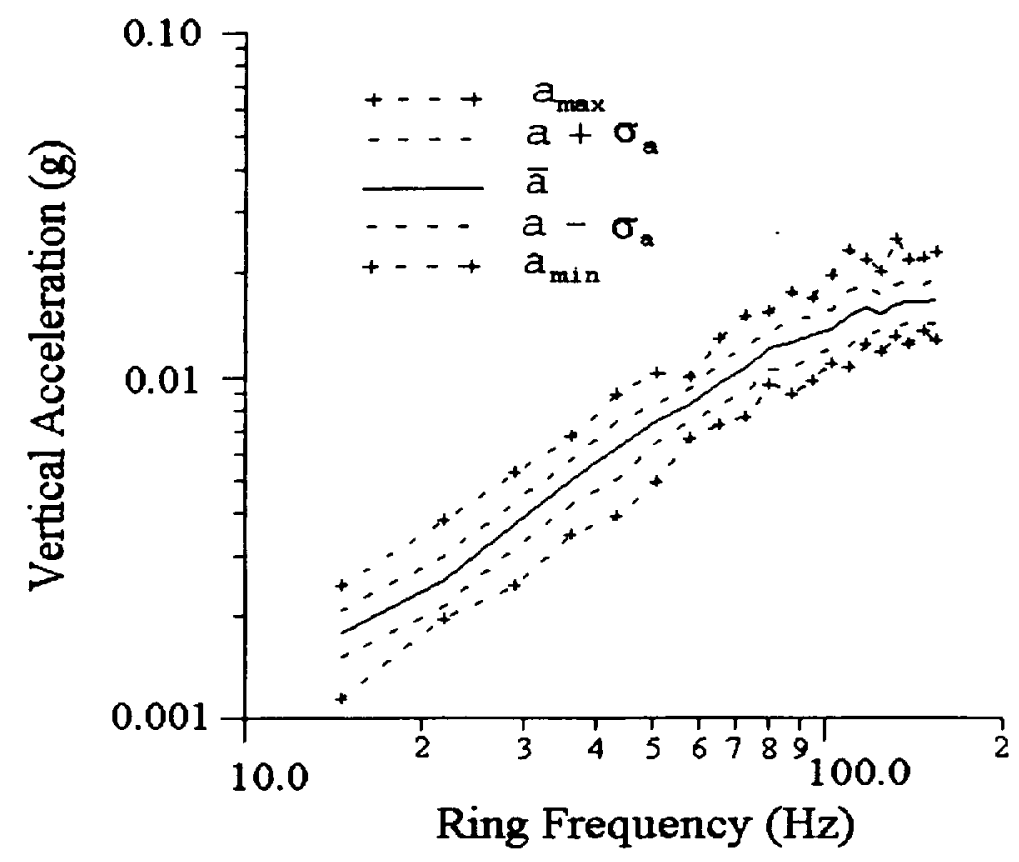

Figure 17: Statistical vertical response spectra of the circular ring without concentrated mass under random base excitation.

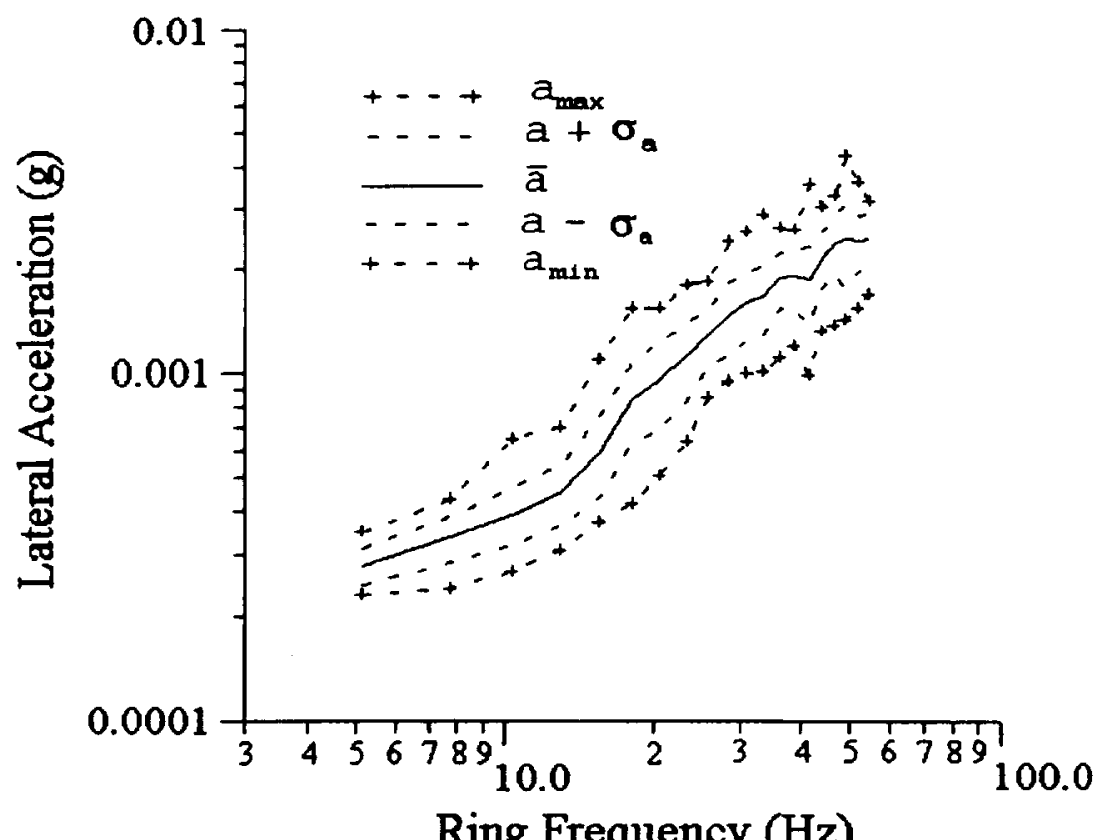

Figure 18: Statistical lateral response spectra of the circular ring without concentrated mass under random base excitation. 


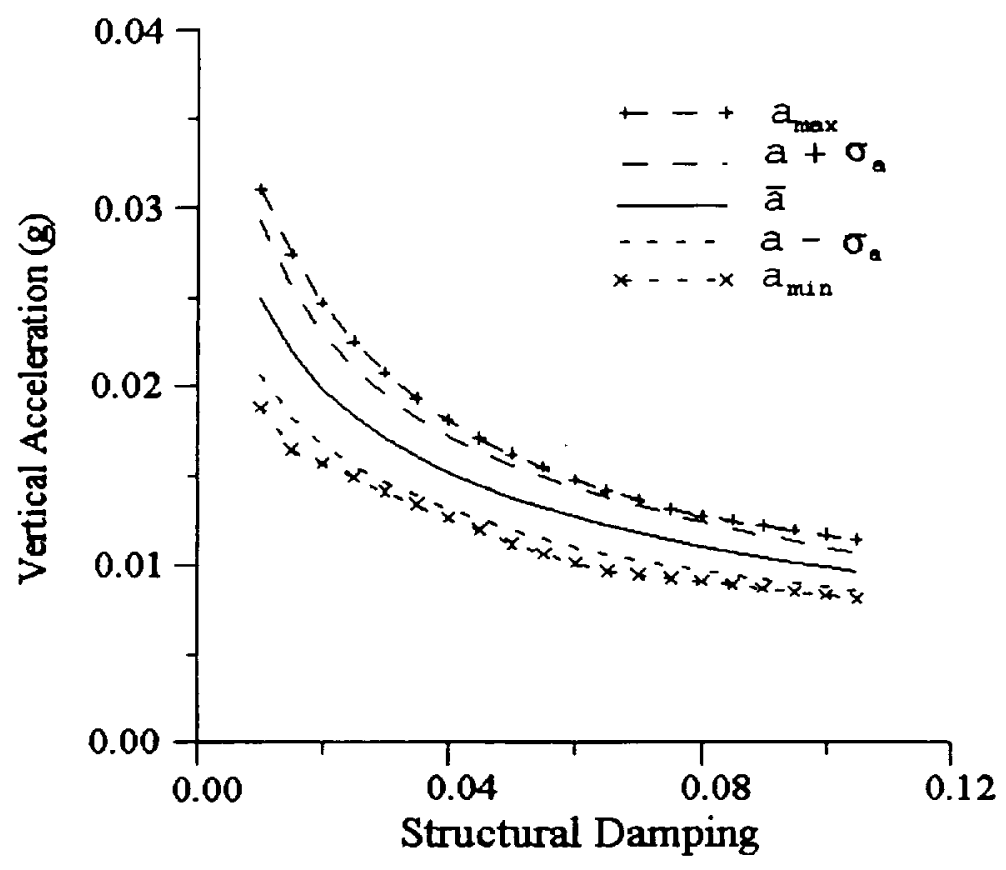

Figure 19: Statistical vertical response spectra of the circular ring as a function of damping under random base excitation.

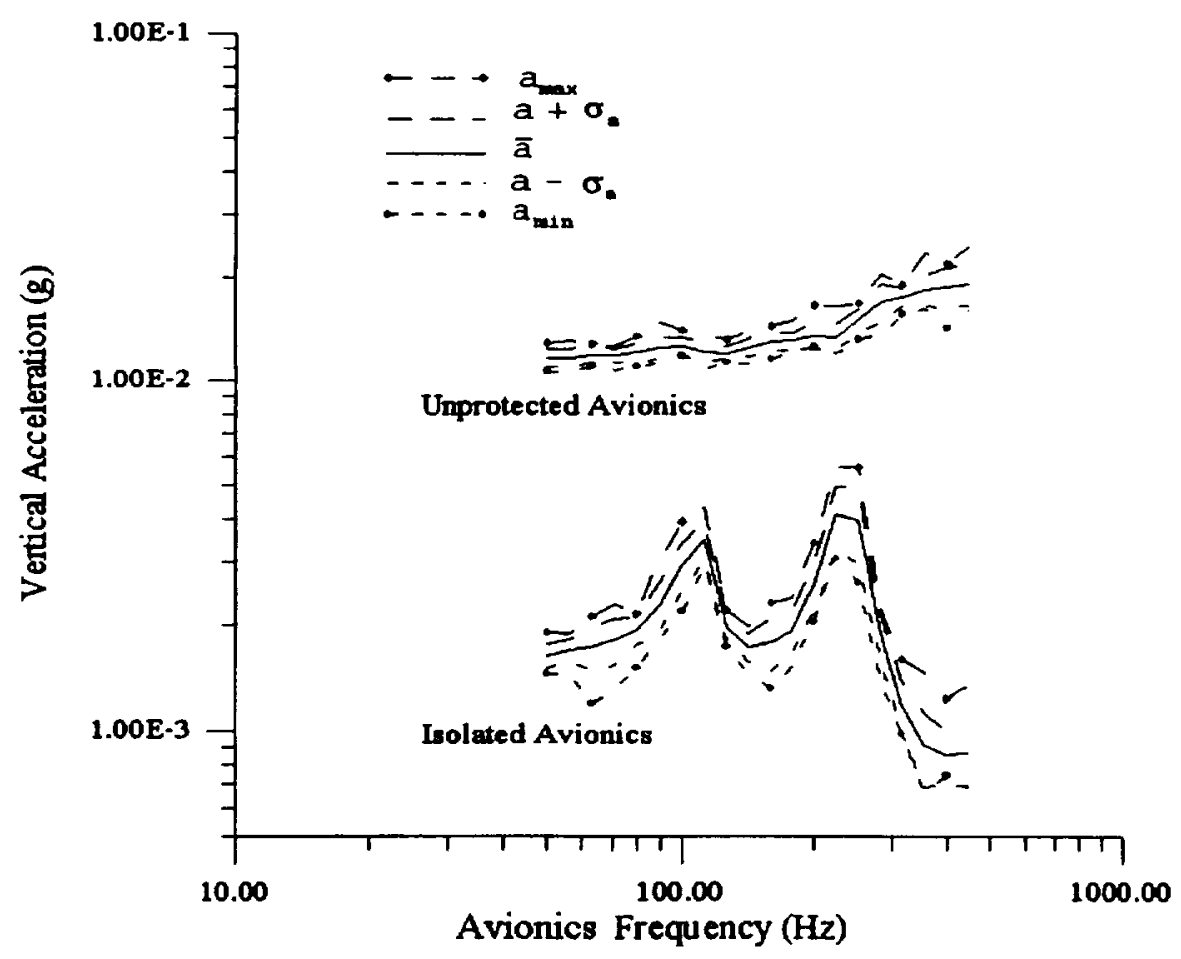

Figure 20: Comparison of vertical response spectra of avionics system with and without the circular ring under random base excitation - rigid mass avionics model. 


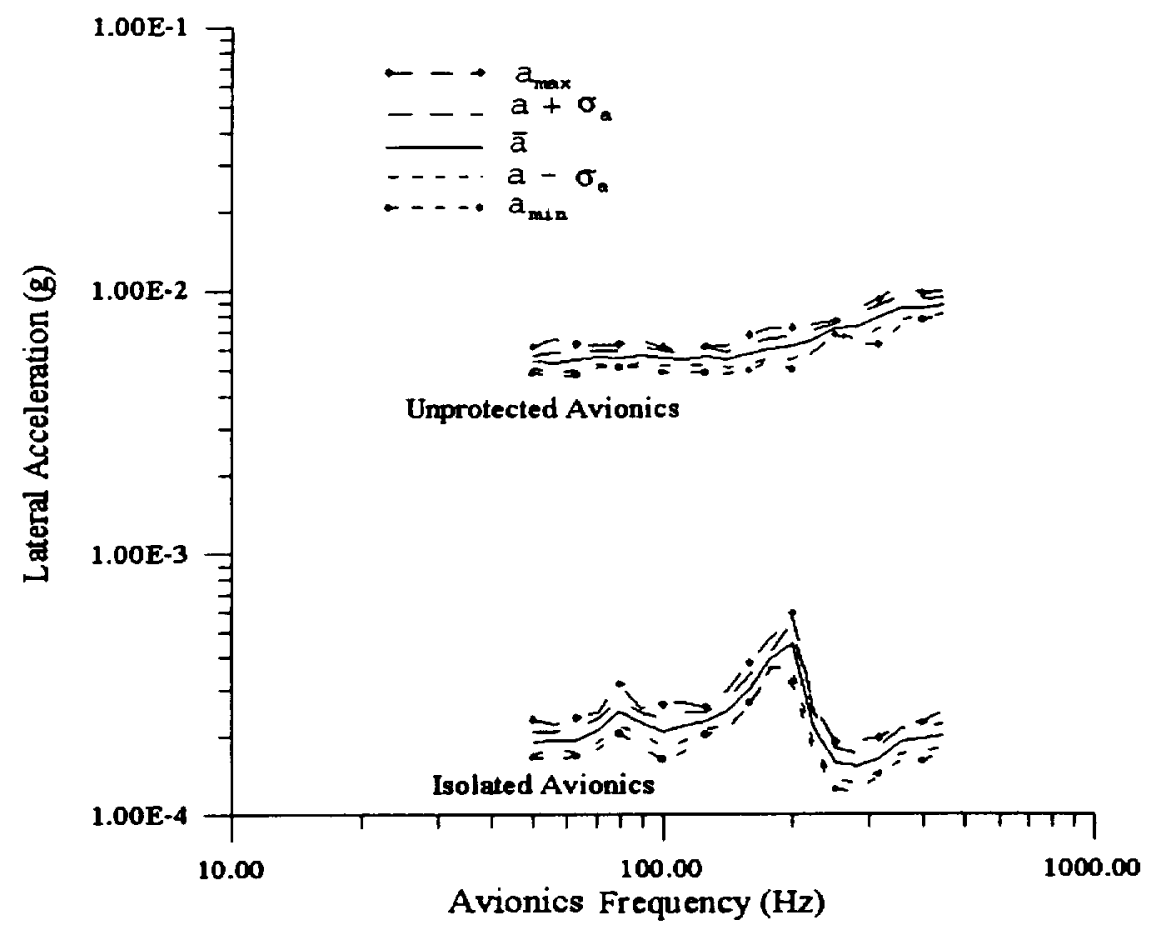

Figure 21: Comparison of lateral response spectra of avionics system with and without the circular ring under random base excitation - rigid mass avionics model.

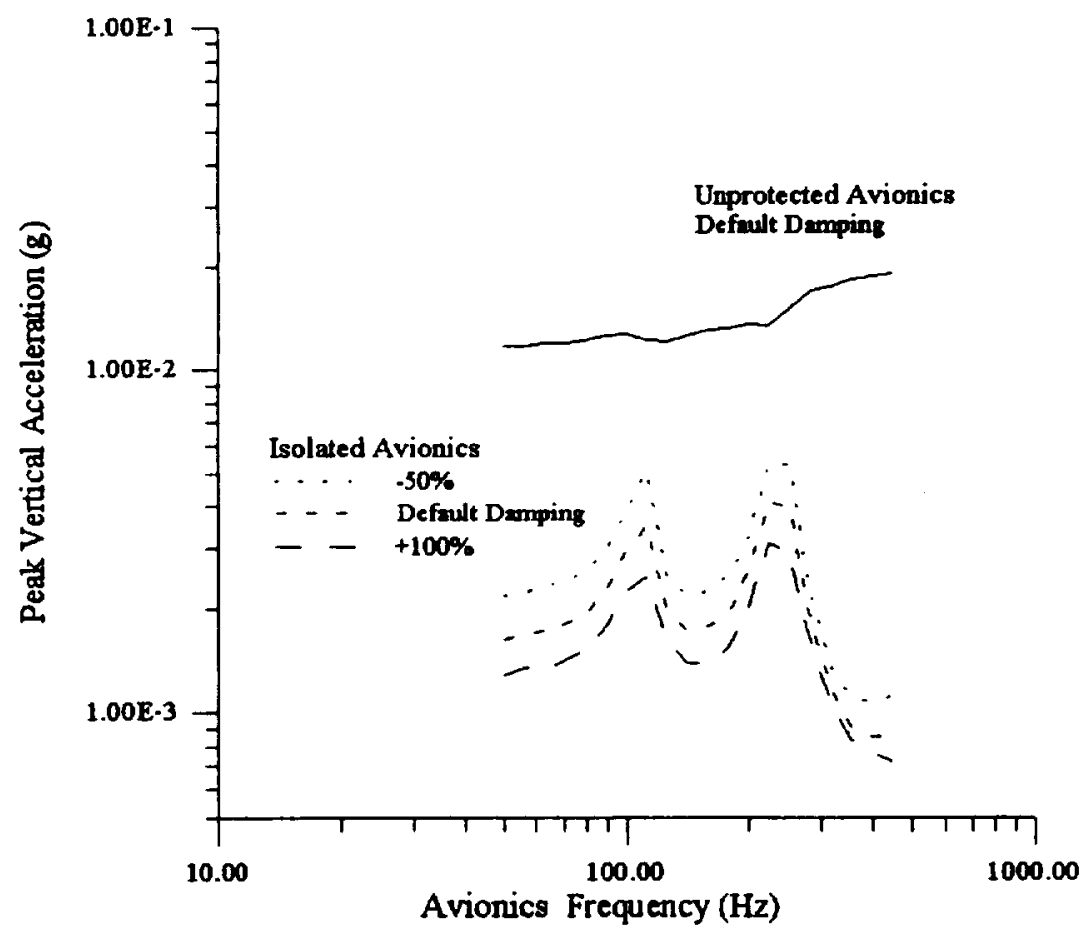

Figure 22: Vertical response spectra of an isolated avionics system with circular ring damping ratio variations as compared to unprotected avionics under random base excitation. 


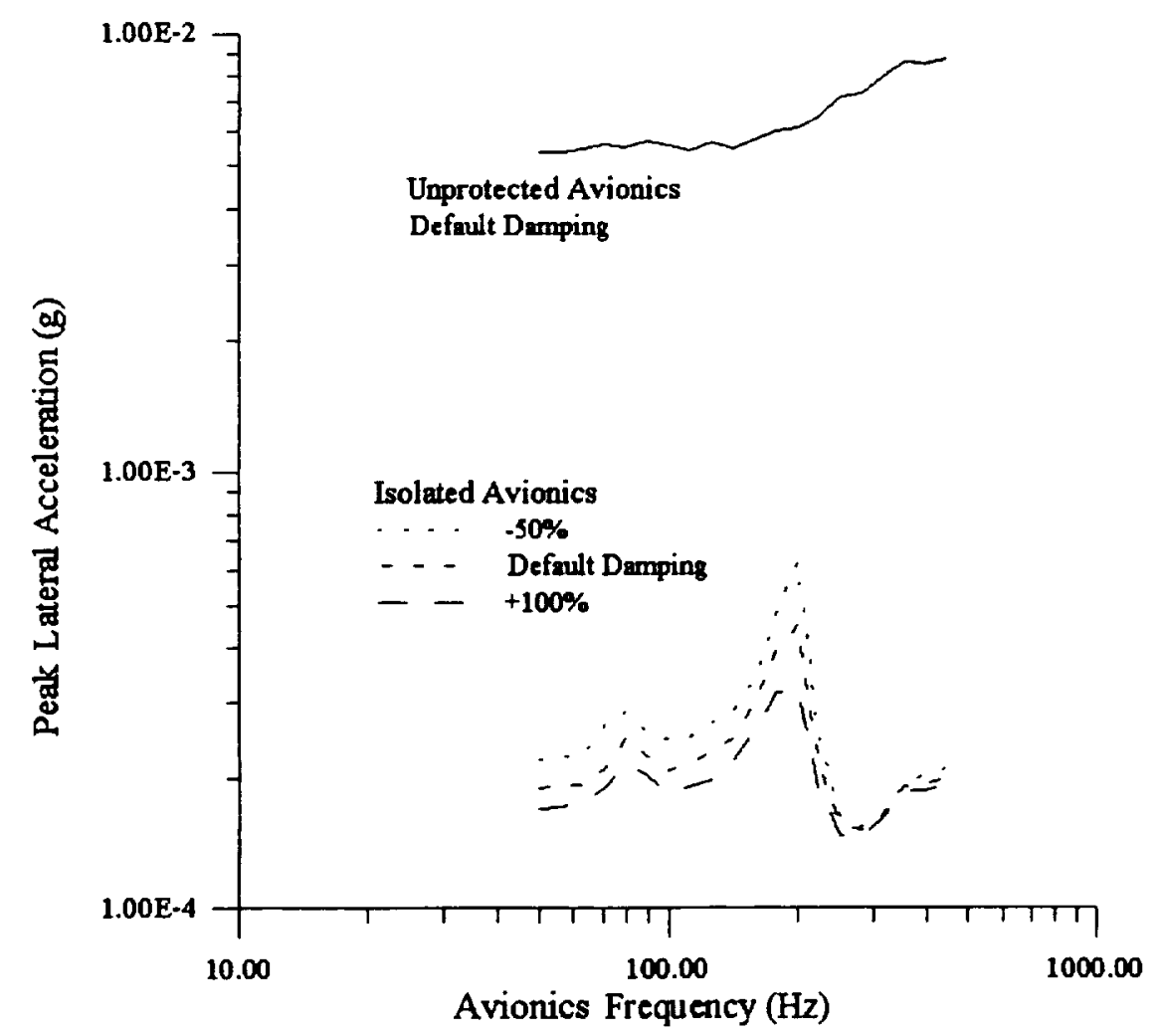

Figure 23: Lateral response spectra of an isolated avionics system with circular ring damping ratio variations as compared to unprotected avionics under random base excitation. 